\title{
On chiral mesons in AdS/CFT
}

\section{Liam McAllister, Paul McGuirk and John Stout}

\author{
Department of Physics, Cornell University, \\ Ithaca, NY 14853, U.S.A. \\ E-mail: mcallister@cornell.edu, mcguirk@cornell.edu, \\ jes554@cornell.edu
}

ABSTRACT: We analyze the spectra of non-chiral and chiral bifundamental mesons arising on intersecting D7-branes in $A d S_{5} \times S^{5}$. In the absence of magnetic flux on the curve of intersection, the spectrum is non-chiral, and the dual gauge theory is conformal in the quenched/probe approximation. For this case we calculate the dimensions of the bifundamental mesonic operators. We then consider magnetization of the D7-branes, which deforms the dual theory by an irrelevant operator and renders the mesons chiral. The magnetic flux spoils the conformality of the dual theory, and induces a D3-brane charge that becomes large in the ultraviolet, where the non-normalizable bifundamental modes are rapidly divergent. An ultraviolet completion is therefore necessary to calculate the correlation functions in the chiral case. On the other hand, the normalizable modes are very well localized in the infrared, leading to new possibilities for local model-building on intersecting D7-branes in warped geometries.

KEYwORDS: Gauge-gravity correspondence, AdS-CFT Correspondence, Intersecting branes models

ARXIV EPRINT: 1311.2577 


\section{Contents}

1 Introduction 1

2 D7-branes in flat space $\quad 4$

2.1 The D7-brane action 4

2.2 Non-chiral modes $\quad 6$

2.3 Chiral modes 8

3 Non-chiral mesons from D7-branes in AdS 11

$\begin{array}{lll}3.1 & \text { Setup and equations of motion } & 12\end{array}$

$\begin{array}{ll}3.2 & \text { The meson spectrum } \\ \end{array}$

4 Chiral mesons from D7-branes in AdS 18

$\begin{array}{lll}4.1 & \text { Setup and equations of motion } & 18\end{array}$

$\begin{array}{ll}4.2 \text { Ultraviolet sensitivity of the correlation functions } & 21\end{array}$

5 Conclusions $\quad 22$

$\begin{array}{ll}\text { A Conventions for fermions } & 24\end{array}$

B Hyperspherical harmonics $\quad 26$

\section{Introduction}

The AdS/CFT correspondence [1-4] is a powerful duality relating conformal field theories (CFTs) in $d$ dimensions to gravitational theories on $(d+1)$-dimensional anti-de Sitter (AdS) spaces. The extension of the duality to include global flavor groups has been wellstudied (see $[5,6]$ for some foundational work) and is well-motivated: it brings the theory closer to phenomenologically viable models, with mesonic bound states serving as prototypes for visible-sector fields. However, to find more realistic models, the flavor group must be extended to a product group, and the resulting mesonic spectrum must be made chiral. Such extensions have been relatively unexplored, and in the present work we report on progress in this direction.

When the gravity side of the duality is a type II string theory, flavor groups are added through the introduction of higher-dimensional $\mathrm{D} p$-branes that fill AdS and wrap compact 
cycles $[6] .{ }^{1}$ The simplest such example is the addition of $F$ D7-branes to type IIB string theory on $A d S_{5} \times S^{5}$, where we take the D7-branes to fill $A d S_{5}$ and wrap an $S^{3}$ of the $S^{5}$. The geometry is supported by $N$ units of D3-brane charge and, without the D7branes, is dual to $\mathcal{N}=4 \mathrm{SU}(N)$ super Yang-Mills. Adding the D7-branes deforms the dual theory to an $\mathcal{N}=2$ gauge theory with a $\mathrm{U}(F)$ flavor group, containing a massless adjoint hypermultiplet as well as a massless quark hypermultiplet that transforms in the bifundamental of $\mathrm{SU}(N) \times \mathrm{U}(F)$. The brane construction makes this clear, as the open string excitations of the D7-branes give rise to a $\mathrm{U}(F)$ gauge theory, and the infinite D7brane worldvolume transverse to the D3-branes results in a vanishing $4 \mathrm{~d}$ coupling for this theory. Open strings stretching between the D7-branes and the D3-branes have the same charges as the quarks in the dual theory. We will work in the standard decoupling limit [1] in which one first takes

$$
g_{\mathrm{s}} \rightarrow 0, \quad N \rightarrow \infty, \quad \lambda_{t} \equiv 4 \pi g_{\mathrm{s}} N \quad \text { fixed },
$$

and then sends the 't Hooft coupling $\lambda_{t}$ to infinity. In this limit, the D3-branes are replaced by their near-horizon backreaction, so that the only open strings are those stretching among the D7-branes. These transform in the adjoint representation of $\mathrm{U}(F)$ and are dual to mesonic operators in the gauge theory.

D7-branes are codimension-two objects, and so their backreaction cannot generally be neglected. Correspondingly, the presence of quarks in the dual gauge theory alters the renormalization group flow, which was trivial before the introduction of flavor. Fortunately, the decoupling limit (1.1) simplifies the situation: if we hold fixed the number of flavors, $F$, while taking the number of colors to be large, then one can consistently neglect the running of quarks in loops. In the dual geometry, many aspects of the D7-brane backreaction scale as $F / N$ and so also vanish in this limit (see [8] and references therein). The flavored gauge theory does have a Landau pole, and so the influence of the quarks on the renormalization group flow cannot be neglected forever, but the scale at which the Landau pole appears grows exponentially with $N / F$. This so-called quenched approximation, in which the running of quarks in loops is neglected, is equivalent to the limit in which the D7-branes are taken as probes of the dual geometry. In what follows, we will take this approximation without further apology.

The introduction of flavor branes opens up significant possibilities for model-building. Dimensional reduction along the angular directions provides a framework for RandallSundrum constructions [9-12] wherein the Standard Model fields propagating in the bulk [13-17] descend from the D7-brane fluctuations as in [18]. Upon compactification, the flavor group on the D7-branes becomes a prototype for the Standard Model gauge group. Of course, the Standard Model gauge group is a product; a corresponding product flavor group results from introducing two separate stacks of D7-branes. The bifundamental fields are then open strings stretching between the stacks, and in order for some of the bifundamentals to be massless, the stacks must intersect.

\footnotetext{
${ }^{1}$ The higher-dimensional D-brane need not fill all of AdS; if the brane is characterized by a minimum distance away from the origin of AdS then the dual quarks are massive [7].
} 
A further challenge is that the Standard Model spectrum is chiral. In the class of constructions considered here, chirality in the $4 \mathrm{~d}$ theory can be induced by introducing magnetic flux on the (noncompact) curve where the D7-branes intersect. Upon compactification to $4 \mathrm{~d}$, the zero modes of the Dirac operator acquire a net chirality set by the amount of quantized magnetic flux.

Yet another difficulty in embedding fully realistic theories into warped backgrounds of string theory is the fact that the Standard Model is not a supersymmetric theory. In geometries that are characterized by a finite infrared scale, such as the well-studied Klebanov-Strassler solution [19], supersymmetry can be broken in a controllable way by the addition of a small number of anti-D3-branes [20]. The resulting geometry [21-29] corresponds to the spontaneous breaking of supersymmetry in the dual field theory [30]. ${ }^{2}$ An alternative is to consider "gluing" the warped geometry to a compact space that does not preserve supersymmetry. The dual field theory is then a non-supersymmetric theory with emergent supersymmetry, as in [31, 32]. Although non-supersymmetric constructions are difficult to control, the filtering provided by the renormalization group means that the influence of the non-supersymmetric bulk, including the effects of moduli stabilization, can be systematically parameterized and incorporated along the lines of [33, 34]. No matter which supersymmetry-breaking mechanism is used, ${ }^{3}$ the resulting geometry is considerably more complex after supersymmetry is broken. We will therefore, in this initial work, focus on supersymmetric D7-brane probes of supersymmetric backgrounds. ${ }^{4}$

In this note, we will consider the non-chiral and chiral bifundamental modes existing at the intersections of probe D7-branes in $A d S_{5} \times S^{5}$. We build up to the chiral, warped case through the simpler example of intersecting D7-branes in flat space (section 2). Although the flat-space analysis of section 2 has appeared elsewhere in the literature (see e.g. [46-49]), a detailed treatment is useful here, because the equations of motion are readily generalized from the simple flat-space case to the $A d S_{5} \times S^{5}$ configuration of primary interest.

The organization of this note is as follows. In section 2 we begin with the simple case of intersecting D7-branes in a flat space background. In section 2.2 we compute the mass spectrum of the bifundamental modes for the case of vanishing magnetic flux, where the spectrum is non-chiral. Then, in section 2.3 we calculate the chiral mass spectrum in a configuration with magnetic flux. Next, in section 3 we consider unmagnetized intersecting D7-branes in $A d S_{5} \times S^{5}$, computing the scaling dimensions of vector-like bifundamental mesonic operators. Finally, in section 4 we add the simplest possible magnetization to the intersecting D7-branes in $A d S_{5} \times S^{5}$, and show that this magnetization makes the calculation of correlation functions untrustworthy without an ultraviolet completion. Concluding remarks are given in section 5, while our conventions and a few technical details appear in the appendices.

\footnotetext{
${ }^{2}$ Some authors have interpreted the singularities of the anti-D3-brane geometry described in [21] as implying that the supersymmetry-breaking state does not exist.

${ }^{3}$ See $[35,36]$ for other interesting proposals.

${ }^{4}$ See, for example, [37-45] for analyses of probe D7-branes in non-supersymmetric backgrounds from the worldvolume and/or worldsheet points of view.
} 


\section{D7-branes in flat space}

As a warm-up to the case of strong warping, we will first review the case of intersecting D7-branes probing unwarped flat space, $\mathbb{R}^{9,1}=\mathbb{R}^{3,1} \times \mathbb{C}^{3}$.

\subsection{The D7-brane action}

As discussed in the introduction, we focus on supersymmetric configurations, and so we take a flat D7-brane probe, which preserves half of the supercharges of flat space. By a choice of orientation and complex structure, the D7-brane worldvolume $\mathcal{W}$ can be taken to be $\mathbb{R}^{3,1} \times \mathbb{C}^{2}$. The light bosonic degrees of freedom resulting from the open-string excitations of the D7-brane consist of the transverse deformations $\Phi^{i}$ and a $\mathrm{U}(1)$ vector potential $A_{1}$. We use this potential to construct a Lorentz-invariant ${ }^{5}$ and supersymmetric magnetic flux $F_{2}=\mathrm{d} A_{1} ;$ such a flux satisfies the self-duality condition [50-52]

$$
F_{2}=\tilde{*}_{4} F_{2},
$$

where $\tilde{*}_{4}$ is the Hodge star built from the metric on $\mathbb{C}^{2}$. The condition (2.1) is equivalent to $F_{2}$ being $(1,1)$ and primitive with respect to the Kähler form induced on $\mathbb{C}^{2}$.

To leading order in the $\alpha^{\prime}$ expansion, the action of the D7-brane in this background is $[53-55]$

$$
S_{\mathrm{D} 7}=-\frac{1}{g_{8}^{2}} \int_{\mathcal{W}} \mathrm{d}^{8} \xi^{\alpha} \sqrt{-\hat{g}}\left\{\frac{1}{2} \hat{g}_{i j} \hat{g}^{\alpha \beta} \partial_{\alpha} \Phi^{i} \partial_{\beta} \Phi^{j}+\frac{1}{4} \hat{g}^{\alpha \beta} \hat{g}^{\gamma \delta} F_{\alpha \gamma} F_{\beta \delta}+\mathrm{i} \bar{\Theta} P_{-}^{\mathrm{D} 7} \hat{g}^{\alpha \beta} \hat{\Gamma}_{\alpha} \partial_{\beta} \Theta\right\},
$$

in which we have omitted a constant term that does not play a role in our analysis. Writing the string tension as $\tau_{\mathrm{F} 1}^{-1}=2 \pi \alpha^{\prime}=\ell_{\mathrm{s}}^{2}$, the $8 \mathrm{~d}$ Yang-Mills coupling is $g_{8}^{-2}=8 \pi^{3} \ell_{\mathrm{s}}^{4} g_{\mathrm{s}}$. Here $\xi^{\alpha}$ are coordinates on the D7-brane, $\hat{g}_{\alpha \beta}$ is the induced worldvolume metric and $\hat{g}_{i j}$ is the transverse metric. $\Theta$ is a 10d double Majorana-Weyl spinor (reviewed in appendix A) that, as in the Green-Schwarz superstring, redundantly encapsulates the fermionic degrees of freedom of the D7-brane. In particular, $\Theta$ is subject to the $\kappa$-symmetry identification

$$
\Theta \sim \Theta+P_{-}^{\mathrm{D} p} \kappa
$$

in which $\kappa$ is an arbitrary Majorana-Weyl double spinor. $P_{-}^{\mathrm{D} 7}$ is given by

$$
P_{-}^{\mathrm{D} 7}=\frac{1}{2}\left(\begin{array}{cc}
1 & -\Gamma_{\mathrm{D} 7}^{-1} \\
-\Gamma_{\mathrm{D} 7} & 1
\end{array}\right),
$$

in which

$$
\Gamma_{\mathrm{D} 7}=\operatorname{dv}_{b} l_{\mathcal{W}}:=\frac{1}{8 !} \hat{\epsilon}_{\alpha_{1} \cdots \alpha_{8}} \hat{\Gamma}^{\alpha_{1} \cdots \alpha_{8}}=-\mathrm{i} \Gamma_{(8)},
$$

where $\epsilon_{\alpha_{1} \cdots \alpha_{8}}$ is the antisymmetric tensor and $\Gamma_{(8)}$ is the $\mathrm{SO}(7,1)$ chirality operator. We use $\kappa$-symmetry to set

$$
\Theta=\left(\begin{array}{l}
\theta \\
0
\end{array}\right) .
$$

\footnotetext{
${ }^{5}$ Here and throughout we will use "Lorentz invariance" to refer to SO $(3,1)$ invariance.
} 
With this choice,

$$
S_{\mathrm{D} 7}=-\frac{1}{g_{8}^{2}} \int_{\mathcal{W}} \mathrm{d}^{8} \xi^{\alpha}\left\{\frac{1}{2} \hat{g}_{i j} \hat{g}^{\alpha \beta} \partial_{\alpha} \Phi^{i} \partial_{\beta} \Phi^{j}+\frac{1}{4} \hat{g}^{\alpha \beta} \hat{g}^{\gamma \delta} F_{\alpha \gamma} F_{\beta \delta}+\frac{\mathrm{i}}{2} \bar{\theta} \hat{g}^{\alpha \beta} \hat{\Gamma}_{\beta} \partial_{\alpha} \theta\right\},
$$

which is the familiar action for maximally supersymmetric $8 \mathrm{~d} \mathrm{U}(1)$ gauge theory.

On a stack of $F$ such D7-branes, the gauge group is enhanced to $\mathrm{U}(F)$, and $A_{\alpha}, \Phi^{i}$, and $\theta$ are promoted to adjoint-valued fields. The leading-order action is determined by gauge-invariance and supersymmetry to be

$$
\begin{aligned}
S_{\mathrm{D} 7}=-\frac{1}{g_{8}^{2}} \int_{\mathcal{W}} \mathrm{d}^{8} \xi^{\alpha} \operatorname{tr}\{ & \frac{1}{2} \hat{g}_{i j} \hat{g}^{\alpha \beta} D_{\alpha} \Phi^{i} D_{\beta} \Phi^{j}+\frac{1}{4} \hat{g}^{\alpha \beta} \hat{g}^{\gamma \delta} F_{\alpha \gamma} F_{\beta \delta}-\frac{1}{4} \hat{g}_{i j} \hat{g}_{k l}\left[\Phi^{i}, \Phi^{k}\right]\left[\Phi^{j}, \Phi^{l}\right] \\
& \left.+\frac{\mathrm{i}}{2} \bar{\theta} \hat{g}^{\alpha \beta} \hat{\Gamma}_{\alpha} D_{\beta} \theta-\frac{1}{2} \bar{\theta} \hat{\Gamma}_{i}\left[\Phi^{i}, \theta\right]\right\}
\end{aligned}
$$

in which tr denotes a trace over gauge indices, $D_{\alpha}$ is a gauge covariant derivative

$$
D_{\alpha}=\partial_{\alpha}-\mathrm{i}\left[A_{\alpha}, \cdot\right]
$$

and $F_{2}=\mathrm{d} A-\mathrm{i} A \wedge A$ is the non-Abelian field strength.

Bifundamental modes arise from strings that stretch between stacks of $\mathrm{D} p$-branes. If the stacks are parallel, then the mass of these modes is proportional to the separation between the branes. Such a configuration still preserves sixteen supercharges and so the action for the bifundamental modes (which provide a full massive vector multiplet) can be fixed by symmetries. Alternatively, the action can be found by Higgsing the theory (2.8). Beginning with a stack of $F_{1}+F_{2}$ D7-branes, the transverse deformations can be treated as $\left(F_{1}+F_{2}\right) \times\left(F_{1}+F_{2}\right)$ matrices with the $i$ th diagonal element corresponding to a transverse deformation of the $i$ th brane. A vacuum expectation value (vev) with the gauge structure

$$
\left\langle\Phi^{i}\right\rangle=\ell_{\mathrm{s}}^{-2}\left(\begin{array}{lll}
X_{1}^{i} \mathbb{I}_{F_{1}} & \\
& X_{2}^{i} \mathbb{I}_{F_{2}}
\end{array}\right)
$$

breaks $\mathrm{U}\left(F_{1}+F_{2}\right) \rightarrow \mathrm{U}\left(F_{1}\right) \times \mathrm{U}\left(F_{2}\right)$ and describes a separation of the branes $\Delta x^{i}=$ $\left|X_{1}^{i}-X_{2}^{i}\right|$. The factor of $\ell_{\mathrm{s}}^{2}$ is introduced so that $\Phi^{i}$ has length dimension -1 . However, this also has the effect of canceling the factors of $\ell_{\mathrm{s}}$ that appear in operators correcting the Yang-Mills action. Therefore, in order to trust this effective field theory, we consider cases where $\Delta x^{i} \ll \ell_{\mathrm{s}}$. Equivalently, if we are to trust the effective field theory description of the modes stretching between the branes, their mass must be less than that of the massive string states that have been integrated out implicitly.

Writing the fluctuations as

$$
\delta \Phi^{i}=\left(\begin{array}{cc}
\phi_{1}^{i} & \phi_{+}^{i} \\
\phi_{-}^{i} & \phi_{2}^{i}
\end{array}\right),
$$

$\phi_{1}^{i}$ and $\phi_{2}^{i}$ transform as adjoints under $\mathrm{U}\left(F_{1}\right)$ and $\mathrm{U}\left(F_{2}\right)$, respectively, while $\phi_{+}^{i}$ and $\phi_{-}^{i}$ are bifundamentals that acquire masses proportional to the separation. For notational simplicity, in what follows we will consider the case $F_{1}=F_{2}=1$, but all of our results generalize easily to higher ranks. 
If, instead of being parallel, the branes intersect, some of the bifundamental modes will become massless. The intersection of two D7-branes is generically six-dimensional, and the long-wavelength description of the bifundamental modes can be given in terms of a $6 \mathrm{~d}$ effective field theory description on this intersection. The $6 \mathrm{~d}$ masses of the bifundamentals depend on the angles formed by the intersection of the branes. However, the vector bifundamentals never become massless, indicating that the 6 d theory is a $\mathrm{U}(1) \times \mathrm{U}(1)$ (rather than the un-Higgsed $U(2)$ ) gauge theory, and that fewer than sixteen supercharges are preserved, since the vector multiplet is split. When the intersection is such that both D7branes fill $\mathbb{R}^{3,1}$ and are holomorphically embedded into $\mathbb{C}^{3}$, at least minimal supersymmetry is preserved [56] and the $6 \mathrm{~d}$ theory includes massless scalars and fermions.

\section{$2.2 \quad$ Non-chiral modes}

In the warped case, the calculation of mass spectra is equivalent to the calculation of scaling dimensions in the dual theory. In this section, we continue our warm-up to the warped case by finding the mass spectrum of non-chiral bifundamental modes in flat space. To this end, we take $z^{I=1,2,3}$ as coordinates on $\mathbb{C}^{3}$ and consider a pair of D7-branes whose embeddings are specified by

$$
\mathrm{D} 7_{1}: z^{3}=t z^{2}, \quad \mathrm{D} 7_{2}: z^{3}=-t z^{2}, \quad t>0 .
$$

Following the discussion in the previous subsection, we can describe this intersection by considering 8d U (2) SYM along $\mathbb{R}^{3,1} \times \mathbb{C}^{2}$ (with $\mathbb{C}^{2}$ spanned by $z^{1}$ and $z^{2}$ ), where the vev for the complexified transverse deformation takes the form ${ }^{6}$

$$
\Phi=q\left(\begin{array}{ll}
z^{2} & \\
& -z^{2}
\end{array}\right),
$$

in which $q=\ell_{\mathrm{s}}^{-2} t$. The bifundamental modes are localized on $\mathbb{R}^{3,1} \times \mathbb{C}$, with $z^{1}$ the coordinate on the curve of intersection (which in this case is simply $\mathbb{C}$ ). For the reasons discussed above, we must take $t \ll 1$ in order to trust the effective field theory. Of course, no matter what the value of $t$, at sufficiently large values of $z^{2}$ the branes will be far apart and so one might worry about stringy corrections to the Yang-Mills action. That is, in addition to (2.8), the worldvolume action contains, for example, operators with the schematic form

$$
\ell_{\mathrm{S}}^{k-4}(\Phi)^{k} \sim\left(\frac{t z^{2}}{\ell_{\mathrm{S}}}\right)^{k-4} \varphi_{ \pm}^{4}+\cdots
$$

which might seem to become important at $z^{2} \sim t^{-1} \ell_{\mathrm{s}}$. However, as we will show below, the bifundamental modes are highly peaked at $z^{2}=0$, and so we anticipate that their physics will be largely insensitive to the corrections at large $z^{2}$.

The configuration just described is supersymmetric, so we can find solutions to the bosonic equations of motion by solving the fermionic equations of motion. Although the intersection is $\mathrm{SO}(5,1)$ symmetric, in anticipation of the magnetization - which preserves only SO $(3,1)$ and which we discuss below - we will make use of the decomposition

\footnotetext{
${ }^{6}$ Similar vevs were utilized in $[46,47]$ to describe brane recombination from non-supersymmetric intersections.
} 
$\mathrm{SO}(9,1) \rightarrow \mathrm{SO}(3,1) \times \mathrm{SO}(6)$, as discussed in appendix A. It is useful to decompose the 10d fermionic mode $\theta$ into modes of different internal chirality (i.e. SO (6) weights)

$$
\theta=\sum_{m=0}^{3}\left\{\psi_{m}\left(\begin{array}{l}
\xi \\
0
\end{array}\right) \otimes \eta_{m}-\psi_{m}^{\dagger}\left(\begin{array}{c}
0 \\
\sigma^{2} \xi^{*}
\end{array}\right) \otimes \tilde{\beta}_{6} \eta_{m}^{*}\right\},
$$

in which $\xi$ is a fixed two-component spinor, $\eta_{m}$ are the constant $\mathrm{SO}(6)$ positive chirality spinors in (A.13), and $\tilde{\beta}_{6}$ is the $\mathrm{SO}(6)$ Majorana matrix. Writing the $\mathrm{U}(1)$ potential as $A_{1}=A_{\mu} \mathrm{d} x^{\mu}+\sum_{a=1}^{2}\left(a_{a} \mathrm{~d} z^{a}+a_{\bar{a}} \mathrm{~d} \bar{z}^{a}\right), \psi_{0}$ is the fermionic partner of $A_{\mu}, \psi_{1,2}$ are the partners of $a_{1}$, and $a_{2}$, and $\psi_{3}$ is the partner of the complexified transverse deformation $\Phi$. Each of the $\psi_{m}$ transforms under the adjoint representation of $\mathrm{U}(2)$ and we write (cf. (2.11))

$$
\psi_{m}=\left(\begin{array}{c}
\psi_{m}^{+} \\
\psi_{m}^{-}
\end{array}\right)
$$

in which we have set the neutral fields $\psi_{m}^{1,2}$ to zero since they are not the modes of interest.

The linearized equation of motion for the fermions in this background is

$$
0=\hat{\Gamma}^{\alpha} \partial_{\alpha} \theta-\mathrm{i} \hat{\Gamma}_{i}\left[\Phi^{i}, \theta\right]
$$

where the transverse fluctuations $\Phi^{i}$ are evaluated on their vev (2.13). From SO $(3,1)$ invariance, we expect that the equation of motion for $A_{\mu}$ should decouple from those of the other bosonic fields, at least for some gauge choice, ${ }^{7}$ and thus we can consistently take $\psi_{0}^{ \pm}$, the superpartner of $A_{\mu}^{ \pm}$, to vanish. When the $4 \mathrm{~d}$ momentum is zero we have

$$
\begin{aligned}
& 0=\bar{\partial}_{\overline{1}} \psi_{1}^{ \pm}-\bar{\partial}_{\overline{2}} \psi_{2}^{ \pm} \mp \mathrm{i} q z^{2} \psi_{3}^{ \pm}, \\
& 0=\partial_{2} \psi_{3}^{ \pm} \mp \mathrm{i} q \bar{z}^{2} \psi_{2}^{ \pm} \\
& 0=\partial_{1} \psi_{3}^{ \pm} \pm \mathrm{i} q \bar{z}^{\overline{2}} \psi_{1}^{ \pm} \\
& 0=\partial_{1} \psi_{2}^{ \pm}+\partial_{2} \psi_{1}^{ \pm}
\end{aligned}
$$

The equations (2.18) also follow from the conditions for supersymmetry [48, 49, 57]. These coupled first-order equations can be turned into largely decoupled second-order equations by taking derivatives. For example, application of $\partial_{1}$ to (2.18a) and substitution of (2.18c) and $(2.18 \mathrm{~d})$ yields

$$
0=\partial_{1} \bar{\partial}_{\overline{1}} \psi_{1}^{ \pm}+\partial_{2} \bar{\partial}_{\overline{2}} \psi_{1}^{ \pm}-q^{2}\left|z^{2}\right| \psi_{1}^{ \pm}
$$

Similarly,

$$
\begin{aligned}
& 0=\partial_{1} \bar{\partial}_{\overline{1}} \psi_{2}^{ \pm}+\partial_{2} \bar{\partial}_{\overline{2}} \psi_{2}^{ \pm} \pm \mathrm{i} q \psi_{3}^{ \pm}-q^{2}\left|z^{2}\right| \psi_{2}^{ \pm}, \\
& 0=\partial_{1} \bar{\partial}_{\overline{1}} \psi_{3}^{ \pm}+\partial_{2} \bar{\partial}_{\overline{2}} \psi_{3}^{ \pm} \mp \mathrm{i} q \psi_{2}^{ \pm}-q^{2}\left|z^{2}\right| \psi_{3}^{ \pm}
\end{aligned}
$$

Using (2.18b), (2.19c) gives an equation for $\psi_{3}^{ \pm}$alone. Writing $\psi_{3}^{ \pm}=\bar{z}^{\overline{2}} \psi_{ \pm}$, we have

$$
0=\partial_{1} \bar{\partial}_{\overline{1}} \psi_{ \pm}+\partial_{2} \bar{\partial}_{\overline{2}} \psi_{ \pm}-q^{2}\left|z^{2}\right| \psi_{ \pm}
$$

\footnotetext{
${ }^{7}$ One such gauge choice is $(2.18 \mathrm{a})$ after simply replacing the fermionic fields with their bosonic partners. See, for example, [49].
} 
Once $\psi_{ \pm}$is determined, $\psi_{1,2,3}^{ \pm}$are easily found.

Equation (2.20) is separable. Performing polar decompositions $z^{a}=r_{a} \mathrm{e}^{\mathrm{i} \phi_{a}}$ and taking the ansatz

$$
\psi_{ \pm}=\mathrm{e}^{\mathrm{i}\left(m_{1} \phi_{1}+m_{2} \phi_{2}\right)} \zeta_{ \pm}\left(r_{1}\right) \sigma_{ \pm}\left(r_{2}\right),
$$

where $m_{i}$ are integers, we have

$$
\begin{aligned}
& 0=\zeta_{ \pm}^{\prime \prime}+\frac{1}{r_{1}} \zeta_{ \pm}^{\prime}-\frac{m_{1}^{2}}{r_{1}^{2}} \zeta_{ \pm}-4 \lambda \zeta_{ \pm}, \\
& 0=\sigma_{ \pm}^{\prime \prime}+\frac{1}{r_{2}} \sigma_{ \pm}^{\prime}-\frac{m_{2}^{2}}{r_{2}^{2}} \sigma_{ \pm}-4 q^{2} r_{2}^{2} \sigma_{ \pm}+4 \lambda \sigma_{ \pm}
\end{aligned}
$$

in which $\lambda$ is a constant to be determined by boundary conditions. Imposing that $\sigma_{ \pm} \rightarrow 0$ as $r_{2} \rightarrow 0$ we find

$$
\begin{aligned}
\zeta_{ \pm}\left(r_{1}\right) & =c_{1} I_{\left|m_{1}\right|}\left(\sqrt{2 \lambda} r_{1}\right)+c_{2} K_{\left|m_{1}\right|}\left(\sqrt{2 \lambda} r_{1}\right), \\
\sigma_{ \pm}\left(r_{2}\right) & =\mathrm{e}^{-q r_{2}^{2}}\left(2 q r_{2}^{2}\right)^{\left|m_{2}\right| / 2} L_{n}^{\left|m_{2}\right|}\left(2 q r_{2}^{2}\right) .
\end{aligned}
$$

in which $L_{\nu}^{\mu}$ are the associated Laguerre polynomials, $I_{\mu}$ and $K_{\mu}$ are the modified Bessel functions of the first and second kinds, and

$$
\lambda=q\left(2 n+\left|m_{2}\right|+1\right) .
$$

Regularity of $\sigma_{ \pm}$requires that $n$ is a non-negative integer. Some of these modes are plotted in figures 1 and 2 .

One may notice that the system (2.18) also admits a zero mode that depends only on $r_{2}$,

$$
\psi_{3}^{ \pm}=\mathrm{e}^{-q r_{2}^{2}}
$$

It is easy to confirm that this gives a solution to (2.20), but this solution is not normalizable with respect to the norm defined by treating (2.20) as a Sturm-Liouville problem. This is a consequence of the fact that the bifundamental modes are more properly encoded by linear combinations of the $\psi_{m}^{ \pm}$rather than by the $\psi_{m}^{ \pm}$themselves [46, 47]. Correspondingly, the measure used in integrating over the $z^{2}$ and $\bar{z}^{\overline{2}}$ directions is not that defined by (2.20) (see [49]). However, aside from this zero mode, the above equations successfully reproduce the spectrum of $6 \mathrm{~d}$ masses $(2.24)$.

\subsection{Chiral modes}

We now consider magnetized intersections since, upon compactification, such a construction gives a chiral $4 \mathrm{~d}$ theory. We will again focus on supersymmetric configurations, which implies that $F_{2}$ must be $(1,1)$ and primitive. Consider first a single D7-brane on $\mathbb{R}^{3,1} \times \mathbb{C}^{2}$. The most general $(1,1)$ flux that can be supported by the D7-brane is

$$
F_{2}=-\frac{\mathrm{i}}{2} f_{1} \mathrm{~d} z^{1} \wedge \mathrm{d} \bar{z}^{\overline{1}}-\frac{\mathrm{i}}{2} f_{2} \mathrm{~d} z^{2} \wedge \mathrm{d} \bar{z}^{\overline{2}}-\frac{\mathrm{i}}{2} g_{1} \mathrm{~d} z^{1} \wedge \mathrm{d} \bar{z}^{\overline{2}}-\frac{\mathrm{i}}{2} g_{2} \mathrm{~d} \bar{z}^{\overline{1}} \wedge \mathrm{d} z^{2} .
$$




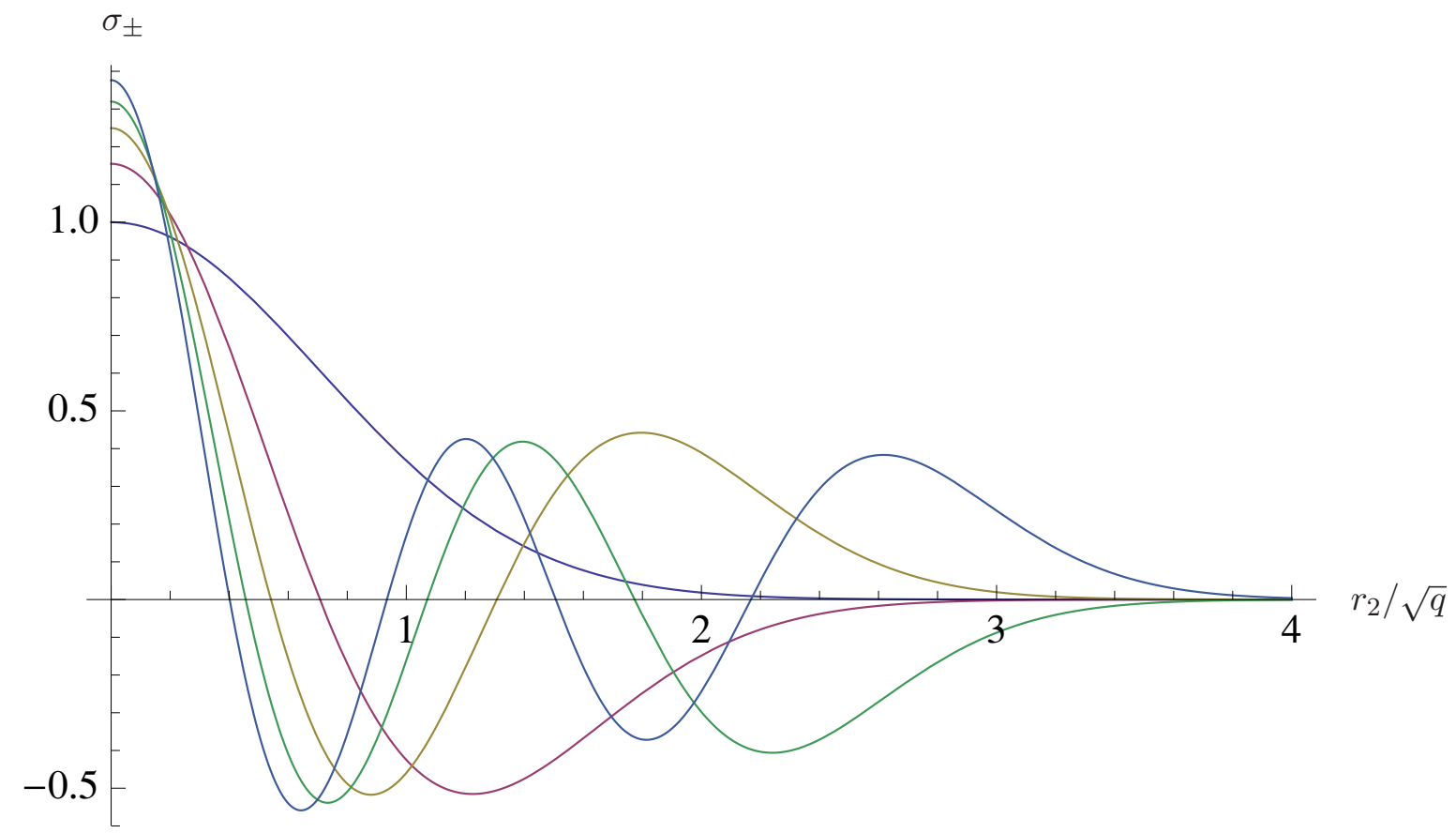

Figure 1. Transverse profiles for the flat space vector-like bifundamental modes $\sigma_{ \pm}$given by (2.23) for $m_{2}=0$ and $n=0$ (the curve with smallest value at $r_{2}=0$ ) through $n=4$ (the curve with the largest value at $r_{2}=0$ ). The solutions have been normalized to the same value using the inner product $\int \mathrm{d} r^{2} f\left(r^{2}\right) g\left(r^{2}\right)$.

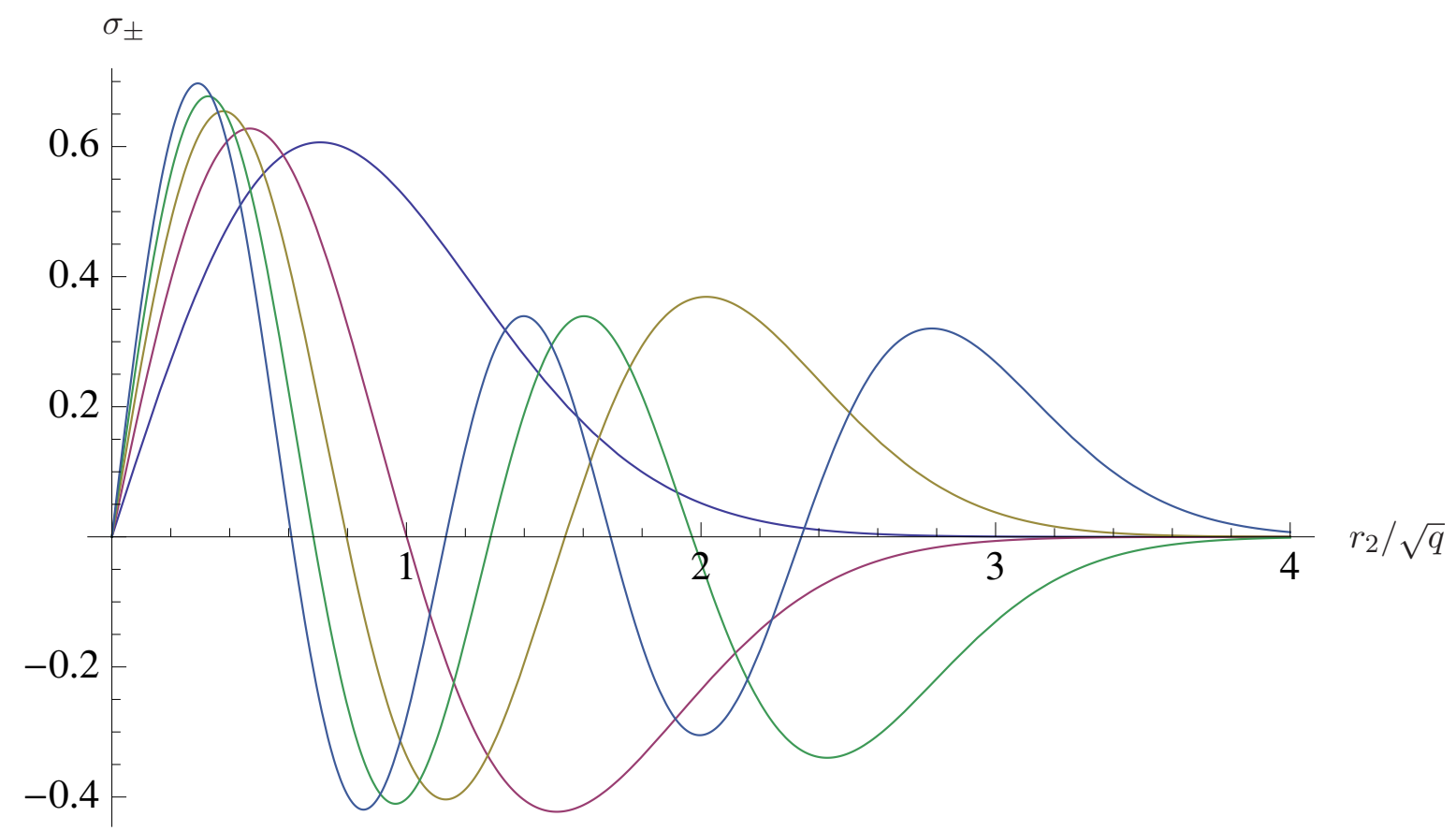

Figure 2. Similar plot as figure 1 except with $m_{2}=1$. 
The $1 \overline{1}$ component will describe the magnetization of the intersection, and so we will look for the simplest configurations with $f_{1} \neq 0$. The Kähler form on $\mathbb{C}^{2}$ is simply

$$
J=-\frac{\mathrm{i}}{2} \sum_{I=1}^{2} \mathrm{~d} z^{I} \wedge \mathrm{d} \bar{z}^{\bar{I}}
$$

and so primitivity imposes $f_{1}=-f_{2}$. The Bianchi identity implies that $f_{1}$ is harmonic,

$$
0=\partial_{1} \bar{\partial}_{1} f_{1}+\partial_{2} \bar{\partial}_{2} f_{1}
$$

In the absence of sources, (2.28) requires that $f_{1}$ is constant. We can then consistently set $g_{1}=g_{2}=0$ and obtain the supersymmetric magnetization

$$
F_{2}=-\mathrm{i} M\left\{\mathrm{~d} z^{1} \wedge \mathrm{d} \bar{z}^{\overline{1}}-\mathrm{d} z^{2} \wedge \mathrm{d} \bar{z}^{\overline{2}}\right\}
$$

Compactification would impose a quantization condition on $M$, but in the non-compact case we can freely take $M$ to be any constant. The above magnetization follows from the gauge choice

$$
A_{1}=-\frac{\mathrm{i}}{2} M\left\{z^{1} \mathrm{~d} \bar{z}^{\overline{1}}-\bar{z}^{\overline{1}} \mathrm{~d} z^{1}-z^{2} \mathrm{~d} \bar{z}^{\overline{2}}+\bar{z}^{\overline{2}} \mathrm{~d} z^{2}\right\} .
$$

To obtain chiral matter, we again consider the intersection of two D7-branes described by the Higgsing (2.13), and choose a magnetization

$$
F_{2}=-M\left(\begin{array}{ll}
1 & \\
& -1
\end{array}\right)\left\{\mathrm{d} z^{1} \wedge \mathrm{d} \bar{z}^{\overline{1}}-\mathrm{d} z^{2} \wedge \mathrm{d} \bar{z}^{\overline{2}}\right\}
$$

The corresponding connection is

$$
A_{1}=-\frac{\mathrm{i}}{2} M\left(\begin{array}{ll}
1 & \\
& -1
\end{array}\right)\left\{z^{1} \mathrm{~d} \bar{z}^{\overline{1}}-\bar{z}^{\overline{1}} \mathrm{~d} z^{1}-z^{2} \mathrm{~d} \bar{z}^{\overline{2}}+\bar{z}^{\overline{2}} \mathrm{~d} z^{2}\right\} .
$$

For simplicity of presentation we will take $M>0$.

With a non-trivial connection, the equation of motion for the fermions becomes

$$
0=\hat{\Gamma}^{\alpha} D_{\alpha} \theta-\mathrm{i} \hat{\Gamma}_{i}\left[\Phi^{i}, \theta\right]
$$

where $D_{\alpha}$ is the gauge-covariant derivative. Following the same decomposition and procedure as for the vector-like case, we again find (2.18) up to the replacements

$$
\begin{array}{ll}
\partial_{1} \psi_{m}^{ \pm} \rightarrow\left(\partial_{1} \pm M \bar{z}^{\overline{1}}\right) \psi_{m}^{ \pm}, & \bar{\partial}_{\overline{1}} \psi_{m}^{ \pm} \rightarrow\left(\bar{\partial}_{\overline{1}} \mp M z^{1}\right) \psi_{m}^{ \pm}, \\
\partial_{2} \psi_{m}^{ \pm} \rightarrow\left(\partial_{2} \mp M \bar{z}^{2}\right) \psi_{m}^{ \pm}, & \bar{\partial}_{\overline{2}} \psi_{m}^{ \pm} \rightarrow\left(\bar{\partial}_{\overline{2}} \pm M z^{2}\right) \psi_{m}^{ \pm}
\end{array}
$$

Again writing $\psi_{3}^{ \pm}=\bar{z}^{\overline{2}} \psi_{ \pm}$, we find

$$
0=\left\{\partial_{1} \bar{\partial}_{\overline{1}}+\partial_{2} \bar{\partial}_{\overline{2}} \pm M\left(\bar{z}^{\overline{1}} \bar{\partial}_{\overline{1}}-z^{1} \partial_{1}-\bar{z}^{2} \bar{\partial}_{\overline{2}}+z^{2} \partial_{2}\right)-M^{2}\left|z^{1}\right|^{2}-\left(M^{2}+q^{2}\right)\left|z^{2}\right|^{2}\right\} \psi_{ \pm} .
$$


Due to the self-duality of the magnetic flux, (2.35) is separable. Again using the polar decomposition $z^{a}=r_{a} \mathrm{e}^{\mathrm{i} \phi_{a}}$ and taking the ansatz (2.21), we find the equations

$$
\begin{aligned}
& 0=\zeta_{ \pm}^{\prime \prime}+\frac{1}{r_{1}} \zeta_{ \pm}^{\prime}-\frac{m_{1}^{2}}{r_{1}^{2}} \zeta_{ \pm}-4 M^{2} r_{1}^{2} \zeta_{ \pm}+\left(-4 \lambda \pm 4 M m_{1}\right) \zeta_{ \pm} \\
& 0=\sigma_{ \pm}^{\prime \prime}+\frac{1}{r_{2}} \sigma_{ \pm}^{\prime}-\frac{m_{2}^{2}}{r_{2}^{2}} \sigma_{ \pm}-4 \kappa^{2} r_{2}^{2} \sigma_{ \pm}+\left(4 \lambda \mp 4 M m_{2}\right) \sigma_{ \pm}
\end{aligned}
$$

in which

$$
\kappa=\sqrt{M^{2}+q^{2}}
$$

and $\lambda$ is again a constant to be determined by boundary conditions. The solutions are

$$
\begin{aligned}
& \zeta_{ \pm}\left(r_{1}\right)=\mathrm{e}^{-M r_{1}^{2}}\left(2 M r_{1}^{2}\right)^{\left|m_{1}\right| / 2}\left\{\mathcal{M}\left(\alpha ; m_{1}+1 ; 2 M r_{1}^{2}\right)+\mathcal{U}\left(\alpha ; m_{1}+1 ; 2 M r_{1}^{2}\right)\right\} \\
& \sigma_{ \pm}\left(r_{2}\right)=\mathrm{e}^{-\kappa r_{2}^{2}}\left(2 \kappa r_{2}^{2}\right)^{\left|m_{2}\right| / 2} L_{n_{2}}^{\left|m_{2}\right|}\left(2 \kappa r_{2}^{2}\right)
\end{aligned}
$$

with

$$
\lambda=\kappa\left(2 n_{2}+\left|m_{2}\right|+1\right) \pm M m_{2} \equiv M\left(2 \alpha-\left|m_{1}\right| \pm m_{1}-1\right),
$$

where the final relation defines $\alpha$. In (2.39), $\mathcal{M}$ and $\mathcal{U}$ are the confluent hypergeometric functions of the first and second kinds, ${ }^{8}$ and regularity requires that $n_{2}$ be a non-negative integer.

The chirality of the spectrum is a consequence of the different behavior of the different charges. It is most easily seen by considering the "missing" zero mode $[49,58]$

$$
\psi_{3}^{ \pm} \sim \mathrm{e}^{-\kappa r_{2}^{2}} \mathrm{e}^{\mp M r_{1}^{2}} h\left(z^{1}\right),
$$

where $h$ is a holomorphic function of $z^{1}$. Since we have taken $M>0$, only the + sector gives rise to normalizable modes, and hence the spectrum is chiral. The fact that $h$ is an arbitrary holomorphic function indicates that there are an infinite number of such chiral modes, as is consistent with the fact that the chiral index, which is proportional to $\int F_{2}$, is divergent. Upon compactification, further conditions are imposed on $h\left(z^{1}\right)$ (see e.g. [58]) and the spectrum becomes finite.

\section{Non-chiral mesons from D7-branes in AdS}

We now consider vector-like mesons arising on intersecting D7-branes in $A d S_{5} \times S^{5}$, building on the groundwork laid in section 2. As discussed in the introduction, the configuration of interest is the gravity dual of $\mathcal{N}=4 \mathrm{SU}(N)$ SYM with a $\mathrm{U}(1) \times \mathrm{U}(1)$ flavor group. The strings stretching between the D7-branes are dual to mesonic operators with charges $( \pm 1, \mp 1)$ under this $\mathrm{U}(1) \times \mathrm{U}(1)$. Our analysis has much in common with the treatment of intersecting D7-branes in weakly warped geometries [49]; however, $A d S_{5} \times S^{5}$ is strongly warped in the sense that no limit of the geometry reproduces a factorized geometry $\mathbb{R}^{3,1} \times X^{6}$, and so we will need to use different techniques to solve the resulting equations of motion.

\footnotetext{
${ }^{8}$ Since the confluent hypergeometric function ${ }_{1} F_{1}(a ; b ; z)$ is not defined when $b=0,-1,-2, \ldots$, we use the regularized version $\mathcal{M}(a ; b ; z)={ }_{1} F_{1}(a ; b ; z) / \Gamma(b)$.
} 


\subsection{Setup and equations of motion}

The metric for $A d S_{5} \times S^{5}$ can be written as a warped product of $\mathbb{R}^{3,1}$ and $\mathbb{C}^{3}$,

$$
\mathrm{d} s_{10}^{2}=\mathrm{e}^{2 \mathcal{A}} \eta_{\mu \nu} \mathrm{d} x^{\mu} \mathrm{d} x^{\nu}+\mathrm{e}^{-2 \mathcal{A}} \mathrm{d} z^{I} \mathrm{~d} \bar{z}^{\bar{I}}, \quad \mathcal{A}=\frac{1}{2} \log \frac{z^{I} \bar{z}^{\bar{I}}}{L^{2}} .
$$

Using hyperspherical coordinates on $\mathbb{C}^{3}=\mathbb{R}^{6}$, this becomes the familiar metric for $A d S_{5} \times S^{5}$,

$$
\mathrm{d} s_{10}^{2}=\frac{R^{2}}{L^{2}} \eta_{\mu \nu} \mathrm{d} x^{\mu} \mathrm{d} x^{\nu}+\frac{L^{2}}{R^{2}} \mathrm{~d} R^{2}+L^{2} \mathrm{~d} s_{S^{5}}^{2},
$$

where $\mathrm{d} s_{S^{5}}^{2}$ is the standard metric on a unit $S^{5}$. The geometry is supported by the 5 form flux

$$
F_{5}=(1+\hat{*}) g_{\mathrm{s}}^{-1} \mathrm{de}^{4 \mathcal{A}} \wedge \operatorname{dvol}_{\mathbb{R}^{3,1}},
$$

where $\hat{*}$ is the 10d Hodge star. In the presence of such flux, the action for a single D7-brane becomes [53-55]

$$
\begin{array}{r}
S_{\mathrm{D} 7}=-\frac{1}{g_{8}^{2}} \int_{\mathcal{W}} \mathrm{d}^{8} \xi^{\alpha} \sqrt{-\hat{g}}\left\{\frac{1}{2} \hat{g}_{i j} \hat{g}^{\alpha \beta} \partial_{\alpha} \Phi^{i} \partial_{\beta} \Phi^{j}+\frac{1}{4} \hat{g}^{\alpha \beta} \hat{g}^{\gamma \delta} F_{\alpha \gamma} F_{\beta \delta}+\mathrm{i} \bar{\Theta} P_{-}^{\mathrm{D} 7} \hat{g}^{\alpha \beta} \hat{\Gamma}_{\alpha} \hat{\nabla}_{\beta} \Theta\right. \\
\left.+\frac{g_{\mathrm{s}}}{8 \cdot 4 !} \hat{\epsilon}^{\alpha_{1} \cdots \alpha_{8}} C_{\alpha_{1} \cdots \alpha_{4}} F_{\alpha_{5} \alpha_{6}} F_{\alpha_{7} \alpha_{8}}+\frac{\mathrm{i} g_{\mathrm{s}}}{16} \bar{\Theta} P_{-}^{\mathrm{D} 7} \hat{g}^{\alpha \beta} \hat{\Gamma}_{\alpha} \hat{H}_{5} \hat{\Gamma}_{\beta}\left(\mathrm{i} \sigma_{2}\right) \Theta\right\},
\end{array}
$$

in which

$$
\hat{F}_{5}=\frac{1}{5 !} F_{M_{1} \cdots M_{5}} \hat{\Gamma}^{M_{1} \cdots M_{5}}
$$

is constructed by contracting all indices of $F_{5}$ with $\hat{\Gamma}$-matrices, and not just those along the worldvolume. If the D7-brane fills $\mathbb{R}^{3,1}$ and a cycle $\mathcal{S}^{4}$ in the other directions, then after $\kappa$-fixing to (2.6) and taking into account the nontrivial spin connection, the fermionic contribution to the action is [59]

$$
S_{\mathrm{D} 7}^{\mathrm{F}}=-\frac{\mathrm{i}}{2 g_{8}^{2}} \int \mathrm{d}^{8} \xi^{\alpha} \sqrt{-\hat{g}} \bar{\theta}\left\{\hat{g}^{\alpha \beta} \hat{\Gamma}_{\alpha} \partial_{\beta}+\frac{1}{2} \hat{g}^{\alpha \beta} \hat{\Gamma}_{\alpha} \partial_{\beta} \mathcal{A}\left(1+2 \hat{\Gamma}_{\mathcal{S}^{4}}\right)\right\} \theta
$$

in which

$$
\hat{\Gamma}_{\mathcal{S}^{4}}=\operatorname{dv} \hat{b} l_{\mathcal{S}^{4}}
$$

is the chirality operator on $\mathcal{S}^{4}$.

In the non-Abelian case, closed-string fields like the warp factor are interpreted as Taylor series in the adjoint-valued transverse deformations, and thus the closed-string fields are themselves adjoint-valued [60]. However, as in the unwarped case, this fact is only important for higher-dimension operators, and can be neglected to leading order in $\ell_{\mathrm{s}}$. Similar terms are expected in the non-Abelian fermionic action, but have not been computed explicitly. However, to leading order in $\ell_{\mathrm{s}}$, supersymmetry and gauge-invariance require that the action take the form $[49,61]$

$$
S_{\mathrm{D} 7}^{\mathrm{F}}=-\frac{\mathrm{i}}{2 g_{8}^{2}} \int \mathrm{d}^{8} \xi^{\alpha} \sqrt{-\hat{g}} \operatorname{tr}\left\{\bar{\theta} \hat{\Gamma}^{\alpha} D_{\alpha} \theta+\frac{1}{2} \bar{\theta} \hat{\Gamma}^{\alpha} \partial_{\alpha} \mathcal{A}\left(1+2 \hat{\Gamma}_{\mathcal{S}^{4}}\right) \theta-\frac{1}{2} \bar{\theta} \hat{\Gamma}_{i}\left[\Phi^{i}, \theta\right]\right\} .
$$


The intersection of two D7-branes satisfying

$$
\mathrm{D} 7_{1}: z^{3}=\mu+t z^{2}, \quad \mathrm{D} 7_{2}: z^{3}=\mu-t z^{2},
$$

is described by

$$
\Phi=\left(\begin{array}{ll}
\ell_{\mathrm{s}}^{-2} \mu+q z^{2} & \\
& \ell_{\mathrm{s}}^{-2} \mu-q z^{2}
\end{array}\right) .
$$

When $\mu=0$, the D7-branes reach the origin of warping and the dual quarks are massless: in the D-brane picture, the D3-branes and D7-branes intersect and the strings stretching between them have zero length. However, when there is a finite separation between the branes, the quarks have a mass proportional to $\mu$. Consequently, the mesonic spectrum becomes gapped [7]. The warp factor is to be evaluated at this vev, but so long as $t$ is sufficiently small, on the D7-brane we can take

$$
\mathcal{A}=\frac{1}{2} \log \frac{\left|z^{1}\right|^{2}+\left|z^{1}\right|^{2}+\mu^{2}}{L^{2}} .
$$

Decomposing $\theta$ as (2.15) and matching terms of internal chirality, we find

$$
\begin{aligned}
0 & =\left(\bar{\partial}_{\overline{1}}-\frac{1}{2} \bar{\partial}_{\overline{1}} \mathcal{A}\right) \psi_{1}^{ \pm}-\left(\bar{\partial}_{\overline{2}}-\frac{1}{2} \bar{\partial}_{\overline{2}} \mathcal{A}\right) \psi_{2}^{ \pm} \mp \mathrm{i} q \mathrm{e}^{-2 \mathcal{A}} z^{2} \psi_{3}^{ \pm}, \\
0 & =\left(\partial_{2}+\frac{3}{2} \partial_{2} \mathcal{A}\right) \psi_{3}^{ \pm} \mp \mathrm{i} q \mathrm{e}^{-2 A} \bar{z}^{2} \psi_{2}^{ \pm} \\
0 & =\left(\partial_{1}+\frac{3}{2} \partial_{1} \mathcal{A}\right) \psi_{3}^{ \pm} \pm \mathrm{i} q \mathrm{e}^{-2 A} \bar{z}^{2} \psi_{1}^{ \pm} \\
0 & =\left(\partial_{1}-\frac{1}{2} \partial_{1} \mathcal{A}\right) \psi_{2}^{ \pm}+\left(\partial_{2}-\frac{1}{2} \partial_{2} \mathcal{A}\right) \psi_{1}^{ \pm}
\end{aligned}
$$

where, as in the flat space analysis of section 2, we have evaluated the equations at zero $4 \mathrm{~d}$ momentum and have set $\psi_{0}^{ \pm}=0$. Taking, as in [59]

$$
\psi_{1,2}^{ \pm}=\mathrm{e}^{\mathcal{A} / 2} \varphi_{1,2}^{ \pm}, \quad \psi_{3}^{ \pm}=\mathrm{e}^{-3 \mathcal{A} / 2} \varphi_{3}^{ \pm},
$$

and finally writing $\varphi_{3}^{ \pm}=\bar{z}^{2} \varphi_{ \pm}$, we find the warped analogue of (2.20)

$$
0=\left\{\partial_{1} \bar{\partial}_{\overline{1}}+\partial_{2} \bar{\partial}_{\overline{2}}-q^{2}\left|z^{2}\right|^{2} \mathrm{e}^{-4 \mathcal{A}}\right\} \varphi_{ \pm} .
$$

Since the warp factor depends on both $z^{1}$ and $z^{2},(3.14)$ is not separable in those variables. However, writing

$$
z^{1}=r \cos \beta \mathrm{e}^{\mathrm{i} \phi_{1}}, \quad z^{2}=r \sin \beta \mathrm{e}^{\mathrm{i} \phi_{2}},
$$

equation (3.14) becomes

$$
0=\left\{\partial_{r}^{2}+\frac{3}{r} \partial_{r}+\frac{1}{r^{2}} \breve{\nabla}^{2}-\frac{4 q^{2} r^{2} L^{4} \sin ^{2} \beta}{\left(r^{2}+\mu^{2}\right)^{2}}\right\} \varphi_{ \pm},
$$


in which

$$
\breve{\nabla}^{2}=\partial_{\beta}^{2}+(\cot \beta-\tan \beta) \partial_{\beta}+\frac{1}{\cos ^{2} \beta} \partial_{\phi_{1}}^{2}+\frac{1}{\sin ^{2} \beta} \partial_{\phi_{2}}^{2}
$$

is the Laplacian on a unit $S^{3}$ (see appendix B).

When $\mu=0,(3.16)$ is completely separable. Indeed, taking

$$
\varphi_{ \pm}=\mathrm{e}^{\mathrm{i}\left(m_{1} \phi_{1}+m_{2} \phi_{2}\right)} f_{ \pm}(r) Q_{ \pm}(\cos 2 \beta)
$$

we find that the radial equation satisfies

$$
0=f_{ \pm}^{\prime \prime}+\frac{3}{r} f_{ \pm}^{\prime}-\frac{\lambda}{r^{2}} f_{ \pm}
$$

while the $\beta$ equation is

$$
0=4\left(1-x^{2}\right) Q_{ \pm}^{\prime \prime}-8 x Q_{ \pm}^{\prime}-\frac{2 m_{1}^{2}}{1+x} Q_{ \pm}-\frac{2 m_{2}^{2}}{1-x} Q_{ \pm}-2 \xi^{2}(1-x) Q_{ \pm}+\lambda Q_{ \pm}
$$

in which $x=\cos 2 \beta$,

$$
\xi^{2} \equiv q^{2} L^{4}=\frac{1}{\pi} t^{2} g_{\mathrm{s}} N
$$

and $\lambda$ is a constant to be determined by boundary conditions. ${ }^{9}$

\subsection{The meson spectrum}

When $\xi=0$, the solutions to (3.20) are the scalar hyperspherical harmonics (see appendix B)

$$
Q_{ \pm}(x)=c(1+x)^{m 1 / 2}(1-x)^{m 2 / 2} P_{\frac{1}{2}\left(\ell-m_{1}-m_{2}\right)}^{\left(m_{2}, m_{1}\right)}(x)
$$

where $P_{n}^{(a, b)}$ are the Jacobi Polynomials, $c$ is the normalization constant (B.12), $\lambda=$ $\ell(\ell+2)$, and the quantum numbers must satisfy the inequalities $0 \leq\left|m_{1}\right|+\left|m_{2}\right| \leq \ell$ and the constraint $\frac{1}{2}\left(\ell-m_{1}-m_{2}\right) \in \mathbb{Z}$.

We have been unable to find analytic solutions to (3.20) when $\xi \neq 0$. However, since (3.20) is an ordinary differential equation, numerical methods readily apply. We implement a spectral method by expanding the unknown solution in terms of the spherical harmonics. The potential term proportional to $\xi$ does not mix modes of different $m_{1}$ and $m_{2}$, so we can accomplish the spectral decomposition by writing

$$
Q(x)=\sum_{\ell} b_{\ell} y_{\ell}(x)
$$

where $y_{\ell}$ are the solutions (3.22) and we have suppressed other indices. Equation (3.20) then becomes

$$
0=\sum_{\ell}\left\{\lambda-\ell(\ell+2)-2 \xi^{2}(1-x)\right\} b_{\ell} y_{\ell}
$$

Using that at fixed $m_{1}$ and $m_{2}$,

$$
\int_{-1}^{1} \mathrm{~d} x y_{\ell} y_{\ell^{\prime}}=\frac{1}{\pi^{2}} \delta_{\ell \ell^{\prime}}
$$

\footnotetext{
${ }^{9}$ Note that in this section and the next, $\lambda$ carries no dimensions, in contrast to the previous section.
} 
and using the recursion relationship (B.13), we can re-express (3.24) as the matrix equation

$$
0=\left[\lambda-\ell(\ell+2)-2 \xi^{2} d_{0}\right] b_{\ell}+2 \xi^{2} d_{-} b_{\ell-2}+2 \xi^{2} d_{+} b_{\ell+2},
$$

with

$$
\begin{aligned}
& d_{0}=\left(1+\frac{m_{2}^{2}-m_{1}^{2}}{\ell(\ell+2)}\right), \\
& d_{-}=\frac{1}{2 \ell} \sqrt{\frac{\left(\ell+m_{1}+m_{2}\right)\left(\ell-m_{1}-m_{2}\right)\left(\ell+m_{1}-m_{2}\right)\left(\ell-m_{1}+m_{2}\right)}{\ell^{2}-1}} \\
& d_{+}=\frac{1}{2(\ell+2)} \sqrt{\frac{\left(\ell+2+m_{1}+m_{2}\right)\left(\ell+2-m_{1}-m_{2}\right)\left(\ell+2+m_{1}-m_{2}\right)\left(\ell+2-m_{1}+m_{2}\right)}{(\ell+1)(\ell+3)}} .
\end{aligned}
$$

Note that even and odd $\ell$ s do not mix, so that this effectively gives two independent matrix equations where the matrices are each tridiagonal.

Solving (3.20) amounts to diagonalization of the matrix defined by (3.26). Unfortunately, because this is an infinite-dimensional matrix, we cannot perform this diagonalization exactly. However, to obtain an estimate of the spectrum, we can truncate the matrix to a finite submatrix. A good rule of thumb in such problems is that including the first $2 n$ modes determines the first $n$ eigenvalues to an accuracy of a few percent [62]. Accurate eigenvalues will be robust against variations in $n$, and our strategy will be to increase the number of modes included until the eigenvalues calculated in this way stabilize. The first few eigenvalues at $m_{1}=m_{2}=0$ resulting from this process are shown in figures 3 and 4 . As $\xi$ increases, the wavefunctions become increasingly localized on the intersection at $\beta=0$, as shown in figure 5 .

Note that when $\xi \ll 1,(3.26)$ immediately yields the perturbative result

$$
\lambda \approx \ell(\ell+2)+2 \xi^{2}\left[1+\frac{m_{2}^{2}-m_{1}^{2}}{\ell(\ell+2)}\right] .
$$

However, since $\xi^{2}=t^{2} g_{\mathrm{s}} N / \pi$, working at $\xi \ll 1$ requires taking $t^{2}$ to be small with respect to the inverse 't Hooft coupling $1 / \lambda$. This limit is of little utility in the present investigation, because we are interested in taking $\lambda \rightarrow \infty$ to suppress $\alpha^{\prime}$ corrections to the leading-order supergravity, cf. (1.1).

If instead $\xi \gg 1$, we find that the spectrum is well-approximated by

$$
\lambda \approx 4 \xi\left(\ell+\left|m_{1}\right|-1\right) .
$$

At large $\xi, \ell$ is no longer a good quantum number, as the intersection badly breaks the rotational symmetry of the $S^{3}$. Correspondingly, the solutions to (3.20) are linear combinations of many different spherical harmonics. However, $m_{1}$ and $m_{2}$ remain good quantum numbers, and so we find it more natural to write the spectrum as

$$
\lambda \approx 4 \xi\left(n+\left|m_{2}\right|+1\right),
$$

where $n=\ell-\left|m_{1}\right|-\left|m_{2}\right|$. 


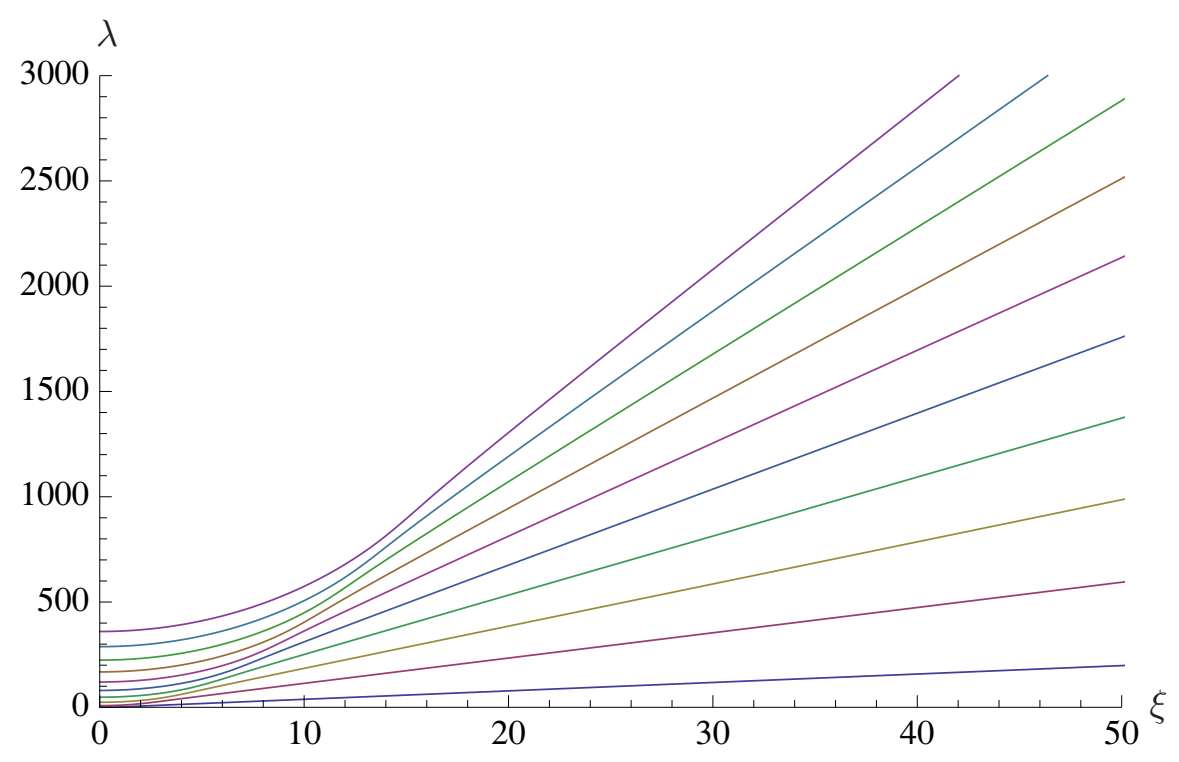

Figure 3. The first few eigenvalues of (3.20) found via spectral methods, for $m_{1}=m_{2}=0$. The growth continues to be linear as $\xi$ increases.

With the eigenvalues of (3.20) in hand, the solution to (3.19) is

$$
f_{ \pm}=c_{1} r^{-1-\sqrt{1+\lambda}}+c_{2} r^{-1+\sqrt{1+\lambda}} .
$$

We can compare the solution (3.31) to the well-known result for a canonically normalized scalar at zero momentum,

$$
\varphi=\varphi_{0} r^{\Delta-4}+\varphi_{1} r^{-\Delta} .
$$

The solution (3.31) does not match the form (3.32), since the transverse deformations are not canonically normalized (see (3.35)). Nevertheless, $\Delta$ can be determined by taking the ratio of the two terms in (3.31), and we find the result

$$
\Delta=2+\sqrt{1+\lambda}
$$

This then gives the approximate expressions

$$
\Delta \approx\left\{\begin{array}{ll}
\ell+3+\frac{\xi^{2}}{1+\ell}\left[1+\frac{\left.m_{2}^{2}-m_{1}^{2}\right]}{\ell(\ell+2)}\right] & \xi \ll 1, . \\
2 \sqrt{\xi\left(n+\left|m_{2}\right|+1\right)} & \xi \gg 1
\end{array} .\right.
$$

The fact that the radial modes are simply power laws is an indication that the dual theory is conformal. Indeed, one can confirm that the $\mu=0$ configuration (3.9) respects the supersymmetry generated by eight supercharges, four of which correspond to the generators of superconformal transformations in the dual theory. Alternatively, when $\mu=0$, the vev (3.10) corresponds to a strictly marginal deformation of the theory. To see this, it suffices to consider the Abelian action (3.4) and examine only the action of the transverse 




Figure 4. The spectrum for $m_{1}=m_{2}=0$ (which requires that $\ell$ be even) for $\xi=0$ (bottom), 25, 50,75 , and 100 (top).

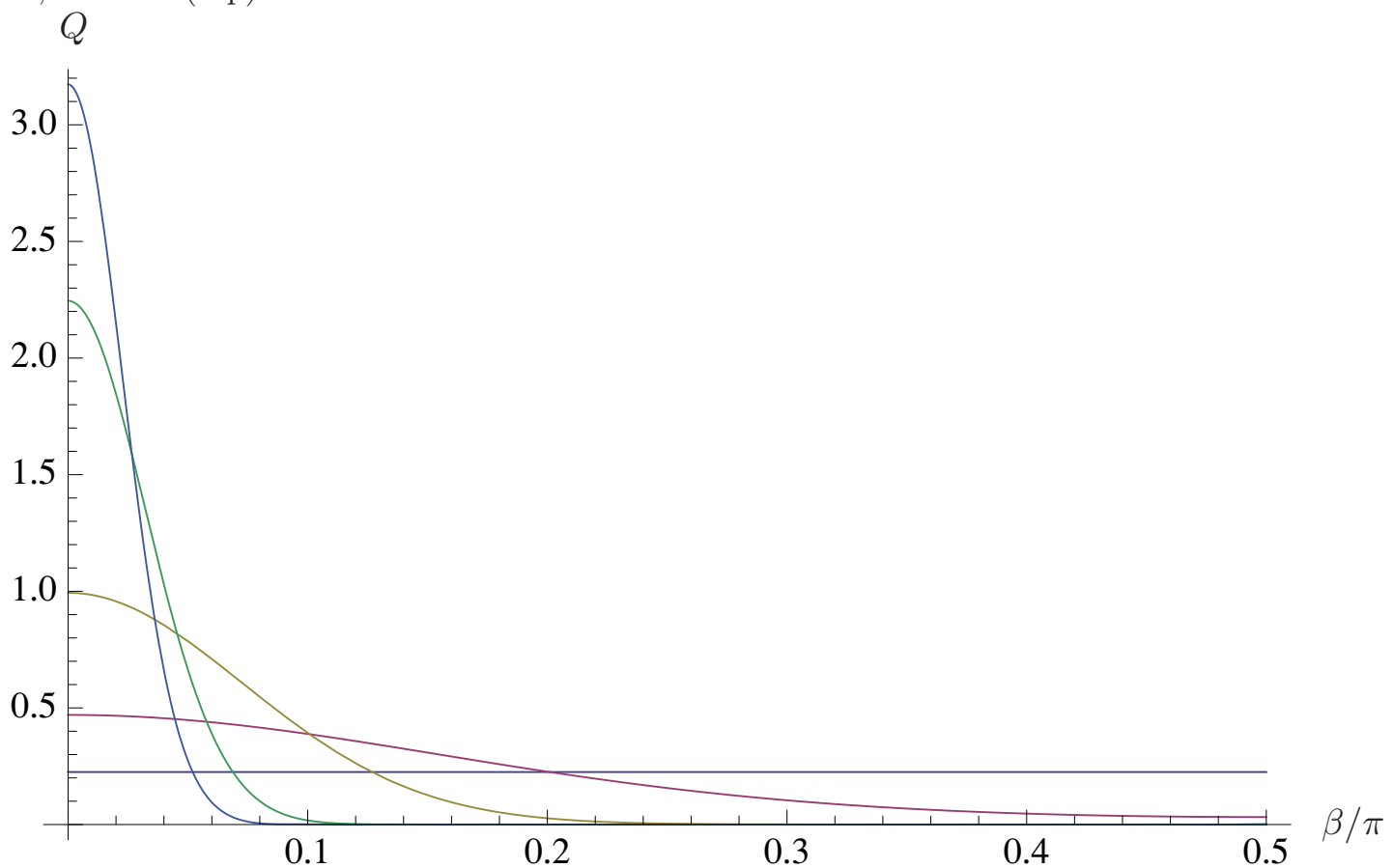

Figure 5. The lowest-lying solutions of (3.20) for $\xi=0,2.5,10,50,100$. When $\xi=0$, the solution is a constant zero mode, but as $\xi$ increases, the profile becomes increasingly peaked at $\beta=0$, the location of the intersection. 
scalars $\Phi^{i}$. Using the complexified field $\Phi$ and expanding in scalar spherical harmonics gives the $5 \mathrm{~d}$ action

$$
S \sim-\int \mathrm{d}^{5} x \sqrt{-g} \sum_{\ell=0}^{\infty}\left\{\frac{L^{2}}{r^{2}} g^{m n} \partial_{m} \Phi_{\ell}^{\dagger} \partial_{n} \Phi_{\ell}+\frac{\ell(\ell+2)}{r^{2}} \Phi_{\ell}^{\dagger} \Phi_{\ell}\right\}
$$

Defining the canonically normalized scalars $\chi_{\ell}=\frac{L}{r} \Phi_{\ell}$ gives

$$
S \sim-\int \mathrm{d}^{5} x \sqrt{-g} \sum_{\ell=0}^{\infty}\left\{g^{m n} \partial_{m} \chi_{\ell}^{\dagger} \partial_{n} \chi_{\ell}+\frac{\ell(\ell+2)-3}{L^{2}} \chi_{\ell}^{\dagger} \chi_{\ell}\right\} .
$$

Using the familiar result

$$
\Delta=2+\sqrt{4+m^{2} L^{2}}
$$

yields

$$
\Delta=\ell+3
$$

With the coordinates of (3.15), the configuration $\Phi=q z^{2}$ can be expressed as

$$
\Phi=q r \sin \beta \mathrm{e}^{\mathrm{i} \phi_{2}}=\frac{q r}{\sqrt{2}} \sqrt{1-\cos 2 \beta} \mathrm{e}^{\mathrm{i} \phi_{2}} .
$$

Comparing to (3.22), the mode (3.39) corresponds to $\ell=1, m_{1}=0, m_{2}=1$, and hence this configuration is the non-normalizable solution of the $\Delta=4$ mode, and so describes a marginal deformation of the dual theory.

\section{Chiral mesons from D7-branes in AdS}

Just as in the flat space case, we can induce chirality into the dual theory through the introduction of a supersymmetric magnetic flux (2.29). However, this magnetic flux will respect only four of the gravity supercharges, and the other four, corresponding to the superconformal charges of the dual theory, will not be preserved. As we shall see, this change has important physical consequences: the calculation of correlation functions will turn out to require counterterms that are super-exponentially sensitive to the ultraviolet completion of the geometry. At the same time, the magnetic flux induces a large amount of D3-brane charge, so that the geometry must be sharply modified in the ultraviolet. In practical terms, this dependence on the ultraviolet behavior presents an obstacle to the calculation of correlation functions. More importantly, it signifies that the magnetization (2.29), and the corresponding appearance of chiral mesons, entails a substantial change in the background.

\subsection{Setup and equations of motion}

We first sketch out the argument regarding the supercharges. A probe D7-brane will preserve the supersymmetry parameterized by a Killing double spinor $\epsilon$ if (cf. (2.4))

$$
P_{-}^{\mathrm{D}} \epsilon=0,
$$


where, with the presence of a magnetic flux $F_{2}, \Gamma_{\mathrm{D} 7}=-\mathrm{i} \Gamma_{(8)} L(F)$ with

$$
L(F)=\sqrt{\frac{\operatorname{det}(\hat{g})}{\operatorname{det}\left(\hat{g}+\ell_{\mathrm{s}}^{2} F\right)}}\left\{1+\frac{\ell_{\mathrm{s}}^{2}}{2} F_{\alpha_{1} \alpha_{2}} \hat{\Gamma}^{\alpha_{1} \alpha_{2}}+\frac{\ell_{\mathrm{s}}^{4}}{8} F_{\alpha_{1} \alpha_{2}} F_{\alpha_{3} \alpha_{4}} \hat{\Gamma}^{\alpha_{1} \alpha_{2} \alpha_{3} \alpha_{4}}\right\} .
$$

The bulk geometry respects the supersymmetry generated by a GKP-like Killing spinor [12], which is independent of the Minkowski coordinates and annihilated by holomorphic $\gamma$ matrices. Moreover, such a Killing spinor obeys (4.1) if $F_{2}$ is $(1,1)$ and self-dual: the $\mathbb{F}_{2}$ term annihilates the Killing spinor, and the 1 and $\not_{2}^{2}$ terms together are canceled by $\sqrt{\operatorname{det}(g+F)}$ (which takes a simple form because $F$ is self-dual). However, the bulk geometry also supports Killing spinors that depend on the Minkowski coordinates in a particular way (see, e.g., [63]). The existence of such spinors is a special feature of anti-de Sitter space, and the supersymmetry transformations they induce are dual to superconformal transformations. Since the special AdS Killing spinors are not preserved by the magnetized D7-brane configuration, we anticipate that conformality will be lost in the dual theory, even in the probe approximation.

We can also understand the loss of conformality from another point of view. The magnetization that gives rise to chirality follows from the connection (2.30) which, using (3.15), can be written as

$$
A_{1}=M r^{2}\left\{-\cos ^{2} \beta \mathrm{d} \phi_{1}+\sin ^{2} \beta \mathrm{d} \phi_{2}\right\}
$$

in which we are still taking $M>0$ for simplicity of presentation. Writing $A_{1}=M r^{2} \omega, \omega$ satisfies the defining equation of a transverse vector spherical harmonic $\varrho$, which takes the general form

$$
\breve{\nabla}^{2} \varrho_{\theta}=-[\ell(\ell+2)-1] \varrho_{\theta}, \quad \breve{\nabla}^{\theta} \varrho_{\theta}=0, \quad \breve{\epsilon}^{\theta \varphi} \psi \breve{\nabla}_{\varphi} \varrho_{\psi}= \pm(\ell+1) \breve{g}^{\theta \psi} \varrho_{\psi}
$$

in which $\breve{g}$ is the metric (B.2) on the unit $S^{3}, \breve{\nabla}$ is the associated Levi-Civita connection, and $\breve{\epsilon}$ is the associated volume form. The mode $\omega$ corresponds to the specific case $\ell=1$, with the positive sign taken in the third equation in (4.4). ${ }^{10}$ Thus, $\omega$ is a transverse vector spherical harmonic [64], and upon dimensional reduction leads to a canonically normalized field with mass $m^{2} L^{2}=12$ (see [7]). This corresponds to an operator of dimension 6 , and $A_{1}$ involves the non-normalizable solution. Hence, the introduction of the magnetic flux deforms the dual theory by an irrelevant operator. This implies that not only is conformality lost in the dual theory, but the theory does not even flow from an ultraviolet fixed point.

\footnotetext{
${ }^{10}$ This sign is independent of the sign in the equation of motion for the bifundamental modes, $(2.18)$.
} 
The addition of this flux modifies the zero-momentum equations to

$$
\begin{aligned}
0 & =\left(\bar{\partial}_{\overline{1}} \mp M z^{1}-\frac{1}{2} \bar{\partial}_{\overline{1}} \mathcal{A}\right) \psi_{1}^{ \pm}-\left(\bar{\partial}_{\overline{2}} \pm M z^{2}-\frac{1}{2} \bar{\partial}_{\overline{2}} \mathcal{A}\right) \psi_{2}^{ \pm} \mp \mathrm{i} q \mathrm{e}^{-2 \mathcal{A}} z^{2} \psi_{3}^{ \pm}, \\
0 & =\left(\partial_{2} \mp M \bar{z}^{\overline{2}}+\frac{3}{2} \partial_{2} \mathcal{A}\right) \psi_{3}^{ \pm} \mp \mathrm{i} q \mathrm{e}^{-2 A} \bar{z}^{\overline{2}} \psi_{2}^{ \pm} \\
0 & =\left(\partial_{1} \pm M \bar{z}^{\overline{1}}+\frac{3}{2} \partial_{1} \mathcal{A}\right) \psi_{3}^{ \pm} \pm \mathrm{i} q \mathrm{e}^{-2 A} \bar{z}^{\overline{2}} \psi_{1}^{ \pm} \\
0 & =\left(\partial_{1} \pm M \bar{z}^{\overline{1}}-\frac{1}{2} \partial_{1} \mathcal{A}\right) \psi_{2}^{ \pm}+\left(\partial_{2} \mp M \bar{z}^{\overline{2}}-\frac{1}{2} \partial_{2} \mathcal{A}\right) \psi_{1}^{ \pm} .
\end{aligned}
$$

Using (3.13), we find

$$
0=\left\{\partial_{1} \bar{\partial}_{\overline{1}}+\partial_{2} \bar{\partial}_{\overline{2}} \pm M\left(\bar{z}^{\overline{1}} \bar{\partial}_{\overline{1}}-z^{1} \partial_{1}-\bar{z}^{2} \bar{\partial}_{\overline{2}}+z^{2} \partial_{2}\right)-M^{2}\left|z^{1}\right|^{2}-\left(M^{2}+\mathrm{e}^{-4 \mathcal{A}} q^{2}\right)\left|z^{2}\right|^{2}\right\} \varphi_{ \pm}
$$

where $\varphi_{ \pm}=\frac{1}{\bar{z}^{2}} \varphi_{ \pm}^{3}$. With the coordinates (3.15), this becomes

$$
\begin{aligned}
0= & \left\{\partial_{r}^{2}+\frac{3}{r} \partial_{r} \pm 4 \mathrm{i} M\left(\partial_{\phi_{1}}-\partial_{\phi_{2}}\right)-4 M^{2} r^{2}\right. \\
& \left.+\frac{1}{r^{2}}\left[\partial_{\beta}^{2}+(\cot \beta-\tan \beta) \partial_{\beta}+\frac{1}{\cos ^{2} \beta} \partial_{\phi_{1}}^{2}+\frac{1}{\sin ^{2} \beta} \partial_{\phi_{2}}^{2}\right]-\frac{4 q^{2} r^{2} L^{4} \sin ^{2} \beta}{\left(r^{2}+\mu^{2}\right)^{2}}\right\} \varphi_{ \pm} .
\end{aligned}
$$

Again, the relative simplicity of this equation is a consequence of the self-duality constraint imposed by supersymmetry. When $\mu=0$, the equation is again separable and it is useful to take the ansatz (3.18). $Q_{ \pm}$satisfies the same eigenvalue problem (3.20) while the radial equation is now

$$
0=f_{ \pm}^{\prime \prime}+\frac{3}{r} f_{ \pm}^{\prime} \mp 4 M\left(m_{1}-m_{2}\right) f_{ \pm}-4 M^{2} r^{2} f_{ \pm}-\frac{\lambda}{r^{2}} f_{ \pm} .
$$

The solutions can be expressed in terms of $\mathcal{M}$ and $\mathcal{U}$, the confluent hypergeometric functions of the first and second kind,

$$
f_{ \pm}=\mathrm{e}^{-M r^{2}} r^{-\nu}\left\{c_{1} \mathcal{M}\left(\mu ; \nu ; 2 M r^{2}\right)+c_{2} \mathcal{U}\left(\mu ; \nu ; 2 M r^{2}\right)\right\}
$$

in which

$$
\nu=1+\sqrt{1+\lambda} \quad \text { and } \quad \mu=\frac{1}{2}\left(\nu \pm\left(m_{1}-m_{2}\right)\right) .
$$

As anticipated, the solutions are not power laws, and so the dual field theory is no longer conformal even in the probe approximation. Furthermore, noting that the dominant asymptotic behavior at $r \rightarrow \infty$ is

$$
\mathcal{M}\left(\mu ; \nu ; 2 M r^{2}\right) \propto e^{2 M r^{2}},
$$

where we have omitted power law factors, we find that the divergent part of (4.9) grows super-exponentially at $r \rightarrow \infty$ :

$$
f_{ \pm} \propto e^{M r^{2}} .
$$




\subsection{Ultraviolet sensitivity of the correlation functions}

To interpret the divergences identified above, it will be helpful to recall the well-established procedure for computing correlation functions in AdS/CFT, focusing on the process of removing divergences of the classical action through the introduction of counterterms, i.e. holographic renormalization (see [65] for a review).

The basic statement of the duality, in the limit (1.1), is the identification of the generating functional of the CFT with the classical supergravity action,

$$
\mathcal{Z}_{\mathrm{CFT}}=\mathrm{e}^{-S_{\text {grav }}}
$$

An operator $\mathcal{O}$ on the field theory side has a corresponding classical field $\varphi$ on the gravity side. If $\mathcal{O}$ is a scalar field, then $\varphi$ also transforms as an $\mathrm{SO}(3,1)$ scalar. The solution for $\varphi$ at large $r$ can be separated into a dominant term and a subdominant term,

$$
\varphi=a_{\mathrm{dom}} \varphi_{\mathrm{dom}}+a_{\mathrm{sub}} \varphi_{\mathrm{sub}}
$$

If the geometry is asymptotically anti-de Sitter space, both the dominant and subdominant terms are power laws at large $r$. Moreover, $a_{\text {dom }}$ is dual to a source term for $\mathcal{O}$, and correlation functions of $\mathcal{O}$ are calculated by taking functional derivatives of $S_{\text {grav }}$ with respect to $a_{\text {dom }}$ and then later taking $a_{\mathrm{dom}} \rightarrow 0$.

For finite $a_{\text {dom }}$, the classical action $S_{\text {grav }}$ is divergent. This can be addressed by adding counterterms to the action: one first regulates the action by cutting off the space at a large but finite radius $r_{\Lambda}$. The terms that diverge as $r_{\Lambda} \rightarrow \infty$ are canceled by adding terms to the supergravity action that are localized on the boundary at $r_{\Lambda}$. Taking $r_{\Lambda} \rightarrow \infty$ then yields a finite action. The power law behavior of solutions in the AdS case means that such counterterms have power-law (and potentially logarithmic) dependence on $r_{\Lambda}$. However, the super-exponential growth (4.12) of the chiral modes requires the introduction of counterterms that have a similar super-exponential dependence on the cutoff. Since the magnetization required to induce chirality deforms the theory by an irrelevant operator, such strong sensitivity is perhaps not surprising.

If the background remained unaltered by magnetization, the structure of counterterms would represent a technically demanding but potentially surmountable challenge to calculating correlation functions. ${ }^{11}$ However, the chirality-inducing magnetic flux sources a large amount of D3-brane charge via the Chern-Simons coupling $\int C_{4} \wedge F_{2} \wedge F_{2}$ : the dissolved D3-brane flux diverges as

$$
\int_{R<\rho} F_{2} \wedge F_{2} \sim M^{2} \rho^{4}
$$

This is comparable to the D3-brane charge of the background when

$$
\rho \sim \frac{N^{1 / 4}}{M^{1 / 2}}
$$

\footnotetext{
${ }^{11}$ For example, as developed in $[66,67]$, it is possible to calculate correlation functions in the KT/KS theory $[19,68]$, even though the theory does not flow from an ultraviolet fixed point.
} 
at which point the influence of this charge on the geometry must be taken into account. A calculation of correlation functions that fails to incorporate this backreaction is not physically meaningful.

One might ask whether a different choice of magnetization (still without a localized source) results in a different conclusion. Supersymmetric fluxes supported on the D7-branes are characterized by scalar hyperspherical harmonics - cf. (2.28) - and so the fluxes grow as $F_{2} \sim r^{j+2} \Omega^{(j)}+r^{j+1} \mathrm{~d} r \wedge \omega^{(j)}$, where $j=0,1,2, \ldots$, and $\Omega^{(j)}$ and $\omega^{(j)}$ are a 2-form and a 1-form on $S^{3}$, respectively. Our analysis of the magnetic flux (2.31) corresponds to the case $j=0$. Other values of $j$ would lead to steeper potentials in (4.6), and so to a greater degree of localization of the bifundamental wavefunctions. However, the charge carried by such flux diverges more quickly than (4.15), growing as $r^{2 j+4}$, and hence the problem of ultraviolet sensitivity is exacerbated.

\section{Conclusions}

In this note we analyzed the spectrum of mesonic operators arising from strings stretching between intersecting D7-branes in $A d S_{5} \times S^{5}$. The dual field theory is an $\mathcal{N}=1$ deformation of maximally supersymmetric $\mathrm{SU}(N)$ SYM, with the addition of a $\mathrm{U}\left(F_{1}\right) \times \mathrm{U}\left(F_{2}\right)$ flavor group, under which the $7-7^{\prime}$ strings transform as bifundamentals. ${ }^{12}$ We considered D7branes with and without magnetic flux on the curve of intersection, finding sharply different results in these two cases.

The intersection of the D7-branes corresponds to a particular adjoint Higgsing of the $\mathrm{U}(2)$ theory arising on coincident D7-branes. In the field theory, the fact that the branes intersect is described by a marginal deformation. If the D7-branes reach the origin of warping, and one furthermore makes the quenched/probe approximation that neglects backreaction of the D7-branes, then the dual theory is conformal. In this case - where magnetization has not yet been incorporated - we computed the spectrum of dual operators. The $7-7^{\prime}$ strings are mixtures of the transverse deformations and the internal components of the gauge field, and as a consequence the equations of motion are difficult to solve analytically. However, conformal symmetry leads to a remarkable simplification of the equations of motion, through which we were able to find numerical solutions. The behavior of the dimensions depends on the value of $\xi \sim \tan \theta \sqrt{g_{\mathrm{s}} N}$, cf. (3.21), where $\theta$ is an angle characterizing the intersection. Approximate spectra are given in (3.34). As expected, the modes are well localized along the intersection of the D7-branes and have power-law behavior along the holographic direction.

We then considered introducing magnetic flux on the curve of intersection, leading to a chiral spectrum in the dual theory. The simplest magnetization corresponds to an irrelevant deformation of the theory, by an operator of dimension $\Delta=6$. As a consequence, the nonnormalizable solutions to the bifundamental equations of motion have super-exponential divergence in the ultraviolet, cf. (4.12). Although the limit (1.1) allows us to neglect the backreaction of the D7-branes themselves, the backreaction of the D3-brane charge induced

\footnotetext{
${ }^{12}$ For notational simplicity only, we limited our discussion to the case $F_{1}=F_{2}=1$, corresponding to a single pair of D7-branes.
} 
by the magnetic flux cannot be neglected. Since the calculation of correlation functions, for example through holographic renormalization, requires the use of the non-normalizable modes, the procedure for calculating the correlation functions is unclear. This is a physical limitation rather than a technical one: the divergence of the D3-brane charge induced by magnetization of noncompact D7-branes signals the need for an ultraviolet completion via compactification. In the dual language, the field theory describing magnetized D7-branes does not flow from an ultraviolet fixed point.

On the other hand, we found that the normalizable modes of the chiral bifundamental mesons are very well localized in the infrared. Indeed, at large $r$,

$$
\mathcal{U}\left(\mu ; \nu ; 2 M r^{2}\right) \sim r^{-\mu},
$$

so that, when $c_{1}=0$ in (4.9), the bifundamental modes exhibit a Gaussian localization,

$$
f_{ \pm} \propto \mathrm{e}^{-M r^{2}}
$$

where we have again omitted power law factors and have chosen $M>0$. Although similar Gaussian peaks appear in flat space (see e.g. [58]), this feature in warped space has the potential to provide a rich playground for model-building. In general, the lack of knowledge of the metric and of related fields often stymies detailed model-building in string compactifications. However, the metrics for infinite families of non-compact (and singular) Calabi-Yau cones are known explicitly. These cones can be used to construct strongly warped geometries that can be attached to compact spaces - see for example the discussion in [12]. Attachment to a compactification modifies the solution in the cone region, by introducing sources for irrelevant perturbations, but these effects can be incorporated systematically, as in [33, 34]. One can therefore build a local model on D3-branes at the apex of the cone, but also take into account bulk effects, including supersymmetry breaking and moduli stabilization. Constructions in this corner of the landscape are limited to some degree by the possible singularities at the apex. An alternative, toward which the present work is a modest advance, is to consider model-building on intersecting magnetized D7-branes. Although the D7-branes will stretch beyond the warped region into the bulk, ${ }^{13}$ we have demonstrated that at least some bifundamental modes are well localized in the infrared. This allows for a combination of the richness of model-building with intersecting D7-branes and the power of local model-building in warped geometries. Although we limited our particular analysis to $A d S_{5} \times S^{5}$, the qualitative result should extend to more general cones and their deformations (though the details, of course, become much more complex).

This localization also implies that although correlation functions are difficult to describe, the mass spectrum of mesons can in principle be calculated with reliable numerical techniques. When the D7-branes move away from the center of $A d S_{5}$, the spectrum of mesons becomes gapped even though, in the quenched approximation, the glueball spectrum is continuous [7]. A standard method of finding the meson mass spectrum in the

\footnotetext{
${ }^{13}$ Indeed, the consistency of embeddings in global models will provide constraints on which models can be built.
} 
gapped case is to calculate the correlation functions and check for the appearance of poles. However, a practical alternative is to find those solutions that satisfy appropriate infrared boundary conditions and are normalizable in the ultraviolet (see, for example, [7, 69]). Because the equation of motion constitutes a Sturm-Liouville problem, this alternative approach leads to a discrete spectrum, and since the solutions are expected to be exponentially convergent, the resulting spectrum would be reliable. On the other hand, once the spectrum becomes gapped the radial and angular parts of the equation of motion no longer separate, even in the unmagnetized case (3.16). This is a significant complication, and so we leave this analysis to future work.

Yet another possibility is to consider alternative magnetizations. The magnetization that we analyzed in this note is the simplest unsourced magnetic flux that is possible in our construction, and other unsourced magnetic fluxes would enhance the bifundamental wavefunction localization that we found, while intensifying the problem of ultraviolet sensitivity. Magnetic flux that is itself localized in the infrared, and produces only normalizable perturbations to the geometry, would require a local source. In particular, it was pointed out in [44] and explicitly shown in [45] that the addition of anti-D3-branes to warped flux backgrounds provides an infrared-localized magnetization. Although the resulting magnetization has a gauge structure that differs from (2.31) - specifically, the induced magnetization is proportional to the identity - this remains an intriguing possibility for future work.

\section{Acknowledgments}

It is a pleasure to thank F. Marchesano and G. Shiu for useful discussions of related topics. This work was supported by the NSF under grant PHY-0757868.

\section{A Conventions for fermions}

In this appendix we summarize our conventions for fermions, many of which follow from [70]. We work with a Weyl basis for the $\mathrm{SO}(9,1) \Gamma$-matrices and make use of the decomposition $\mathrm{SO}(9,1) \rightarrow \mathrm{SO}(3,1) \times \mathrm{SO}(6)$. For $\mathrm{SO}(3,1)$ we take

$$
\gamma^{0}=\left(\begin{array}{c}
\mathbb{I}_{2} \\
-\mathbb{I}_{2}
\end{array}\right), \quad \gamma^{i=1,2,3}=\left(\begin{array}{c}
\sigma^{i} \\
\sigma^{i}
\end{array}\right),
$$

in which $\sigma^{i}$ are the Pauli matrices

$$
\sigma^{1}=\left(\begin{array}{ll}
0 & 1 \\
1 & 0
\end{array}\right), \quad \sigma^{2}=\left(\begin{array}{cc}
0 & -\mathrm{i} \\
\mathrm{i} & 0
\end{array}\right), \quad \sigma^{3}=\left(\begin{array}{cc}
1 & 0 \\
0 & -1
\end{array}\right) .
$$

For $\mathrm{SO}(2 k+1,1)$, we take the chirality matrix to be

$$
\gamma_{(2 k+2)}=\mathrm{i}^{-k} \mathrm{dv} b \mathrm{l}_{\mathbb{R}^{2 k+1,1}},
$$

where $\operatorname{dvol}_{M}$ is the volume element on $M$

$$
\operatorname{dvol}_{M}=\frac{1}{d !} \epsilon_{M_{1} \cdots M_{d}} \mathrm{~d} x^{M_{1}} \wedge \cdots \wedge \mathrm{d} x^{M_{d}},
$$


in which $\epsilon_{01 \cdots(d-1)}=\sqrt{-\operatorname{det} g}$. For $\mathbb{R}^{3,1}$,

$$
\gamma_{(4)}=-\mathrm{i} \gamma^{0} \gamma^{1} \gamma^{2} \gamma^{3}=\left(\begin{array}{ll}
\mathbb{I}_{2} & \\
& -\mathbb{I}_{2}
\end{array}\right) .
$$

The 4d Majorana matrix is

$$
\beta_{4}=\gamma_{(4)} \gamma^{2}=\left(\sigma^{-\sigma^{2}}\right)
$$

For SO (6), we define

$$
\begin{array}{ll}
\tilde{\gamma}^{4}=\sigma^{1} \otimes \mathbb{I}_{2} \otimes \mathbb{I}_{2}, & \tilde{\gamma}^{7}=\sigma^{2} \otimes \mathbb{I}_{2} \otimes \mathbb{I}_{2}, \\
\tilde{\gamma}^{5}=\sigma^{3} \otimes \sigma^{1} \otimes \mathbb{I}_{2}, & \tilde{\gamma}^{8}=\sigma^{3} \otimes \sigma^{2} \otimes \mathbb{I}_{2}, \\
\tilde{\gamma}^{6}=\sigma^{3} \otimes \sigma^{3} \otimes \sigma^{1}, & \tilde{\gamma}^{9}=\sigma^{3} \otimes \sigma^{3} \otimes \sigma^{2} .
\end{array}
$$

For $\mathrm{SO}(2 k+4)$, the chirality operator is

$$
\gamma_{(2 k+4)}=\mathrm{i}^{-k} \mathrm{dv} b \mathrm{l}_{\mathbb{R}^{2 k+4}},
$$

and so

$$
\tilde{\gamma}_{(6)}=-\mathrm{i} \tilde{\gamma}^{1} \cdots \tilde{\gamma}^{6}=\sigma^{3} \otimes \sigma^{3} \otimes \sigma^{3} .
$$

The Majorana matrix is

$$
\tilde{\beta}_{6}=\tilde{\gamma}^{7} \tilde{\gamma}^{8} \tilde{\gamma}^{9}=\sigma^{2} \otimes \mathrm{i} \sigma^{1} \otimes \sigma^{2} .
$$

We will make use of a complex structure

$$
z^{I}=x^{3+I}+\mathrm{i} x^{4+I} .
$$

Defining

$$
\sigma^{ \pm}=\frac{1}{2}\left(\sigma^{1} \pm \mathrm{i} \sigma^{2}\right)
$$

we have

$$
\begin{array}{ll}
\tilde{\gamma}^{1}=2 \sigma^{+} \otimes \mathbb{I}_{2} \otimes \mathbb{I}_{2}, & \tilde{\gamma}^{\overline{1}}=2 \sigma^{-} \otimes \mathbb{I}_{2} \otimes \mathbb{I}_{2}, \\
\tilde{\gamma}^{2}=2 \sigma^{3} \otimes \sigma^{+} \otimes \mathbb{I}_{2}, & \tilde{\gamma}^{\overline{2}}=2 \sigma^{3} \otimes \sigma^{-} \otimes \mathbb{I}_{2}, \\
\tilde{\gamma}^{3}=2 \sigma^{3} \otimes \sigma^{3} \otimes \sigma^{+}, & \tilde{\gamma}^{\overline{3}}=2 \sigma^{3} \otimes \sigma^{3} \otimes \sigma^{-} .
\end{array}
$$

We can construct a basis of positive chirality spinors by first defining

$$
\eta_{+}=\left(\begin{array}{l}
1 \\
0
\end{array}\right), \quad \eta_{-}=\left(\begin{array}{l}
0 \\
1
\end{array}\right) .
$$

The positive chirality spinors are then

$$
\eta_{0}=\eta_{+++}, \quad \eta_{1}=\eta_{+--}, \quad \eta_{2}=\eta_{-+-}, \quad \eta_{3}=\eta_{--+},
$$


in which

$$
\eta_{\epsilon_{1} \epsilon_{2} \epsilon_{3}}=\eta_{\epsilon_{1}} \otimes \eta_{\epsilon_{2}} \otimes \eta_{\epsilon_{3}} .
$$

Note that $\sigma^{ \pm} \eta_{ \pm}=0$, so that $\eta_{+++}$is annihilated by all contravariant holomorphic $\tilde{\gamma}$-matrices.

Finally, we construct the $\mathrm{SO}(9,1) \Gamma$-matrices by

$$
\hat{\Gamma}^{\mu}=\gamma^{\mu} \otimes \mathbb{I}_{8}, \quad \hat{\Gamma}^{m}=\gamma_{(6)} \otimes \tilde{\gamma}^{m} .
$$

The chirality and Majorana matrices are

$$
\begin{aligned}
\hat{\Gamma}_{(10)} & =\hat{\Gamma}^{0} \hat{\Gamma}^{1} \cdots \hat{\Gamma}^{9}=-\gamma_{(4)} \otimes \tilde{\gamma}_{(6)}, \\
\hat{B}_{10} & =\hat{\Gamma}^{2} \hat{\Gamma}^{7} \hat{\Gamma}^{8} \hat{\Gamma}^{9}=-\beta_{4} \otimes \tilde{\beta}_{6} .
\end{aligned}
$$

We will make use of 32-component Majorana-Weyl spinors satisfying

$$
\hat{\Gamma}_{(10)} \theta=-\theta, \quad \hat{B}_{10} \theta=\theta^{*} .
$$

An example of such a spinor is

$$
\theta=\left(\begin{array}{l}
\xi \\
0
\end{array}\right) \otimes \eta-\left(\begin{array}{c}
0 \\
\sigma^{2} \xi^{*}
\end{array}\right) \otimes \tilde{\beta}_{6} \eta^{*}
$$

where $\tilde{\gamma}_{(6)} \eta=+\eta$.

We will also make use of double spinors built from pairs of 10d Majorana-Weyl spinors

$$
\Theta=\left(\begin{array}{l}
\theta^{1} \\
\theta^{2}
\end{array}\right)
$$

where both $\theta^{1}$ and $\theta^{2}$ satisfy (A.17). $\hat{\Gamma}$-matrices act on double spinors as

$$
\hat{\Gamma}^{M} \Theta=\left(\begin{array}{c}
\hat{\Gamma}^{M} \theta^{1} \\
\hat{\Gamma}^{M} \theta^{2}
\end{array}\right),
$$

while explicit Pauli matrices act to mix the elements of the double spinor. For example,

$$
\sigma^{1}\left(\begin{array}{l}
\theta^{1} \\
\theta^{2}
\end{array}\right)=\left(\begin{array}{l}
\theta^{2} \\
\theta^{1}
\end{array}\right)
$$

\section{B Hyperspherical harmonics}

In this appendix we review a few properties of the hyperspherical harmonics on $S^{3}$. A useful parametrization of $S^{3}$ is via the usual embedding of $S^{3}$ into $\mathbb{R}^{4}, \zeta^{i} \zeta^{i}=1$, where $\zeta^{1} \ldots \zeta^{4}$ are coordinates on $\mathbb{R}^{4}$. We take (as in, for example, [71])

$$
\begin{array}{rlrl}
\zeta^{1} & =r \cos \beta \cos \phi_{1}, & \zeta^{2} & =r \sin \beta \cos \phi_{2}, \\
\zeta^{3} & =r \cos \beta \sin \phi_{1}, & \zeta^{4}=r \sin \beta \sin \phi_{2},
\end{array}
$$


with $\beta \in\left[0, \frac{\pi}{2}\right]$ and $\phi_{a} \in[0,2 \pi)$. The induced metric on $S^{3}$ is

$$
\mathrm{d} s_{S^{3}}^{2}=\breve{g}_{\theta \varphi} \mathrm{d} y^{\theta} \mathrm{d} y^{\varphi}=\mathrm{d} \beta^{2}+\cos ^{2} \beta \mathrm{d} \phi_{1}^{2}+\sin ^{2} \beta \mathrm{d} \phi_{2}^{2} .
$$

The volume of $S^{3}$ is

$$
\mathcal{V}_{S^{3}}=\int_{0}^{2 \pi} \mathrm{d} \phi_{1} \int_{0}^{2 \pi} \mathrm{d} \phi_{2} \int_{0}^{\pi / 2} \mathrm{~d} \beta \sin \beta \cos \beta=2 \pi^{2} .
$$

The scalar spherical harmonics satisfy the eigenvalue problem

$$
\breve{\nabla}^{2} \mathcal{Y}=\frac{\partial^{2} \mathcal{Y}}{\partial \beta^{2}}+(\cot \beta-\tan \beta) \frac{\partial \mathcal{Y}}{\partial \beta}+\frac{1}{\cos ^{2} \beta} \frac{\partial^{2} \mathcal{Y}}{\partial \phi_{1}^{2}}+\frac{1}{\sin ^{2} \beta} \frac{\partial^{2} \mathcal{Y}}{\partial \phi_{2}^{2}}=-\lambda \mathcal{Y}
$$

Taking the ansatz

$$
\mathcal{Y}=\mathrm{e}^{\mathrm{i}\left(m_{1} \phi_{1}+m_{2} \phi_{2}\right)} y(\cos 2 \beta)
$$

gives

$$
0=4\left(1-x^{2}\right) y^{\prime \prime}-8 x y^{\prime}-\frac{2 m_{1}^{2}}{1+x} y-\frac{2 m_{2}^{2}}{1-x} y+\lambda y,
$$

in which $x=\cos 2 \beta$. Imposing Neumann conditions so that a zero mode is admitted, the solutions are given in terms of Jacobi polynomials $P_{r}^{(a, b)}$,

$$
\begin{aligned}
\mathcal{Y}_{\ell, m_{1}, m_{2}}\left(\beta, \phi_{1}, \phi_{2}\right)=c_{\ell, m_{1}, m_{2}} \mathrm{e}^{\mathrm{i}\left(m_{1} \phi_{1}+m_{2} \phi_{2}\right)}(1+\cos 2 \beta)^{m_{1} / 2} \times \\
\times(1-\cos 2 \beta)^{m_{2} / 2} P_{r}^{\left(m_{2}, m_{1}\right)}(\cos 2 \beta),
\end{aligned}
$$

in which $r=\frac{1}{2}\left(\ell-m_{1}-m_{2}\right)$. For these to be non-vanishing regular solutions, $r$ must be an integer and

$$
0 \leq\left|m_{1}\right|+\left|m_{2}\right| \leq \ell
$$

These solutions satisfy

$$
\breve{\nabla}^{2} \mathcal{Y}=-\ell(\ell+2) \mathcal{Y}
$$

and the condition (B.8) gives the expected degeneracy of $(\ell+1)^{2}$ (see, for example, [64]).

The Jacobi polynomials are orthogonal in the sense that

$$
\int_{-1}^{1} \mathrm{~d} x(1-x)^{a}(1+x)^{b} P_{r}^{(a, b)} P_{s}^{(a, b)}=\frac{2^{a+b+1}}{2 r+a+b+1} \frac{(a+r) !(b+r) !}{r !(a+b+r) !} \delta_{r s} .
$$

Therefore the normalization condition

$$
\int \operatorname{dvol}_{S^{3}} \mathcal{Y}_{\ell, m_{1}, m_{2}}^{*} \mathcal{Y}_{\ell^{\prime}, m_{1}^{\prime}, m_{2}^{\prime}}=\delta_{\ell^{\prime} \ell} \delta_{m_{1}^{\prime} m_{1}} \delta_{m_{2}^{\prime} m_{2}}
$$

is satisfied by taking

$$
c_{\ell, m_{1}, m_{2}}=\frac{1}{\pi} \sqrt{\frac{\ell+1}{2^{m_{1}+m_{2}+1}} \frac{\left[\frac{1}{2}\left(\ell+m_{1}+m_{2}\right)\right] !\left[\frac{1}{2}\left(\ell-m_{1}-m_{2}\right)\right] !}{\left[\frac{1}{2}\left(\ell+m_{1}-m_{2}\right)\right] !\left[\frac{1}{2}\left(\ell-m_{1}+m_{2}\right)\right] !} .}
$$

The Jacobi polynomials satisfy the useful recursion relationship

$$
\begin{aligned}
x P_{r}^{(a, b)}(x)= & \frac{2(a+r)(b+r)}{(a+b+2 r)(a+b+2 r+1)} P_{r-1}^{(a, b)}(x) \\
& +\frac{2(r+1)(a+b+r+1)}{(a+b+2 r+1)(a+b+2 r+2)} P_{r+1}^{(a, b)}(x) \\
& +\frac{b^{2}-a^{2}}{(a+b+2 r)(a+b+2 r+2)} P_{r}^{(a, b)}(x) .
\end{aligned}
$$


Open Access. This article is distributed under the terms of the Creative Commons Attribution License (CC-BY 4.0), which permits any use, distribution and reproduction in any medium, provided the original author(s) and source are credited.

\section{References}

[1] J.M. Maldacena, The large- $N$ limit of superconformal field theories and supergravity, Adv. Theor. Math. Phys. 2 (1998) 231 [Int. J. Theor. Phys. 38 (1999) 1113] [hep-th/9711200] [INSPIRE].

[2] E. Witten, Anti-de Sitter space and holography, Adv. Theor. Math. Phys. 2 (1998) 253 [hep-th/9802150] [INSPIRE].

[3] S. Gubser, I.R. Klebanov and A.M. Polyakov, Gauge theory correlators from noncritical string theory, Phys. Lett. B 428 (1998) 105 [hep-th/9802109] [INSPIRE].

[4] O. Aharony, S.S. Gubser, J.M. Maldacena, H. Ooguri and Y. Oz, Large-N field theories, string theory and gravity, Phys. Rept. 323 (2000) 183 [hep-th/9905111] [INSPIRE].

[5] O. Aharony, A. Fayyazuddin and J.M. Maldacena, The large- $N$ limit of $N=2, N=1$ field theories from three-branes in F-theory, JHEP 07 (1998) 013 [hep-th/9806159] [INSPIRE].

[6] A. Karch and E. Katz, Adding flavor to AdS/CFT, JHEP 06 (2002) 043 [hep-th/0205236] [INSPIRE].

[7] M. Kruczenski, D. Mateos, R.C. Myers and D.J. Winters, Meson spectroscopy in AdS/CFT with flavor, JHEP 07 (2003) 049 [hep-th/0304032] [INSPIRE].

[8] C. Núñez, A. Paredes and A.V. Ramallo, Unquenched flavor in the gauge/gravity correspondence, Adv. High Energy Phys. 2010 (2010) 196714 [arXiv: 1002.1088] [INSPIRE].

[9] L. Randall and R. Sundrum, A large mass hierarchy from a small extra dimension, Phys. Rev. Lett. 83 (1999) 3370 [hep-ph/9905221] [INSPIRE].

[10] H.L. Verlinde, Holography and compactification, Nucl. Phys. B 580 (2000) 264 [hep-th/9906182] [INSPIRE].

[11] B.R. Greene, K. Schalm and G. Shiu, Warped compactifications in $M$ and F theory, Nucl. Phys. B 584 (2000) 480 [hep-th/0004103] [INSPIRE].

[12] S.B. Giddings, S. Kachru and J. Polchinski, Hierarchies from fluxes in string compactifications, Phys. Rev. D 66 (2002) 106006 [hep-th/0105097] [INSPIRE].

[13] H. Davoudiasl, J. Hewett and T. Rizzo, Bulk gauge fields in the Randall-Sundrum model, Phys. Lett. B 473 (2000) 43 [hep-ph/9911262] [INSPIRE].

[14] A. Pomarol, Gauge bosons in a five-dimensional theory with localized gravity, Phys. Lett. B 486 (2000) 153 [hep-ph/9911294] [INSPIRE].

[15] S. Chang, J. Hisano, H. Nakano, N. Okada and M. Yamaguchi, Bulk standard model in the Randall-Sundrum background, Phys. Rev. D 62 (2000) 084025 [hep-ph/9912498] [INSPIRE].

[16] Y. Grossman and M. Neubert, Neutrino masses and mixings in nonfactorizable geometry, Phys. Lett. B 474 (2000) 361 [hep-ph/9912408] [INSPIRE].

[17] T. Gherghetta and A. Pomarol, Bulk fields and supersymmetry in a slice of AdS, Nucl. Phys. B 586 (2000) 141 [hep-ph/0003129] [INSPIRE]. 
[18] T. Gherghetta and J. Giedt, Bulk fields in $A d S_{5}$ from probe D7 branes, Phys. Rev. D 74 (2006) 066007 [hep-th/0605212] [INSPIRE].

[19] I.R. Klebanov and M.J. Strassler, Supergravity and a confining gauge theory: duality cascades and $\chi_{S B}$ resolution of naked singularities, JHEP 08 (2000) 052 [hep-th/0007191] [INSPIRE].

[20] S. Kachru, R. Kallosh, A.D. Linde and S.P. Trivedi, De Sitter vacua in string theory, Phys. Rev. D 68 (2003) 046005 [hep-th/0301240] [INSPIRE].

[21] I. Bena, M. Graña and N. Halmagyi, On the existence of meta-stable vacua in Klebanov-Strassler, JHEP 09 (2010) 087 [arXiv:0912.3519] [INSPIRE].

[22] O. DeWolfe, S. Kachru and M. Mulligan, A gravity dual of metastable dynamical supersymmetry breaking, Phys. Rev. D 77 (2008) 065011 [arXiv:0801.1520] [INSPIRE].

[23] P. McGuirk, G. Shiu and Y. Sumitomo, Non-supersymmetric infrared perturbations to the warped deformed conifold, Nucl. Phys. B 842 (2011) 383 [arXiv:0910.4581] [INSPIRE].

[24] A. Dymarsky, On gravity dual of a metastable vacuum in Klebanov-Strassler theory, JHEP 05 (2011) 053 [arXiv:1102.1734] [INSPIRE].

[25] I. Bena, G. Giecold, M. Graña, N. Halmagyi and S. Massai, On metastable vacua and the warped deformed conifold: analytic results, Class. Quant. Grav. 30 (2013) 015003 [arXiv: 1102.2403] [INSPIRE].

[26] I. Bena, G. Giecold, M. Graña, N. Halmagyi and S. Massai, The backreaction of anti-D3 branes on the Klebanov-Strassler geometry, JHEP 06 (2013) 060 [arXiv:1106.6165] [INSPIRE].

[27] S. Massai, A comment on anti-brane singularities in warped throats, arXiv:1202.3789 [INSPIRE].

[28] I. Bena, M. Graña, S. Kuperstein and S. Massai, Anti-D3's - Singularl to the bitter end, Phys. Rev. D 87 (2013) 106010 [arXiv:1206.6369] [InSPIRE].

[29] A. Dymarsky and S. Massai, Uplifting the baryonic branch: a test for backreacting anti-D3-branes, arXiv:1310.0015 [INSPIRE].

[30] S. Kachru, J. Pearson and H.L. Verlinde, Brane/flux annihilation and the string dual of a nonsupersymmetric field theory, JHEP 06 (2002) 021 [hep-th/0112197] [INSPIRE].

[31] T. Gherghetta and A. Pomarol, The standard model partly supersymmetric, Phys. Rev. D 67 (2003) 085018 [hep-ph/0302001] [INSPIRE].

[32] R. Sundrum, SUSY splits, but then returns, JHEP 01 (2011) 062 [arXiv:0909.5430] [INSPIRE].

[33] D. Baumann, A. Dymarsky, S. Kachru, I.R. Klebanov and L. McAllister, D3-brane potentials from fluxes in AdS/CFT, JHEP 06 (2010) 072 [arXiv: 1001.5028] [INSPIRE].

[34] S. Gandhi, L. McAllister and S. Sjors, A toolkit for perturbing flux compactifications, JHEP 12 (2011) 053 [arXiv:1106.0002] [INSPIRE].

[35] S. Kachru, D. Simic and S.P. Trivedi, Stable non-supersymmetric throats in string theory, JHEP 05 (2010) 067 [arXiv:0905.2970] [INSPIRE].

[36] A. Dymarsky and S. Kuperstein, Non-supersymmetric conifold, JHEP 08 (2012) 033 [arXiv:1111.1731] [INSPIRE]. 
[37] P.G. Cámara, L. Ibáñez and A. Uranga, Flux-induced SUSY-breaking soft terms on D7-D3 brane systems, Nucl. Phys. B 708 (2005) 268 [hep-th/0408036] [INSPIRE].

[38] D. Lüst, S. Reffert and S. Stieberger, Flux-induced soft supersymmetry breaking in chiral type IIB orientifolds with D3/D7-branes, Nucl. Phys. B 706 (2005) 3 [hep-th/0406092] [INSPIRE].

[39] D. Lüst, S. Reffert and S. Stieberger, MSSM with soft SUSY breaking terms from D7-branes with fluxes, Nucl. Phys. B 727 (2005) 264 [hep-th/0410074] [INSPIRE].

[40] C. Burgess et al., Warped Supersymmetry Breaking, JHEP 04 (2008) 053 [hep-th/0610255] [INSPIRE].

[41] D. Lüst, F. Marchesano, L. Martucci and D. Tsimpis, Generalized non-supersymmetric flux vacua, JHEP 11 (2008) 021 [arXiv:0807.4540] [INSPIRE].

[42] P. McGuirk, G. Shiu and Y. Sumitomo, Holographic gauge mediation via strongly coupled messengers, Phys. Rev. D 81 (2010) 026005 [arXiv:0911.0019] [INSPIRE].

[43] P. McGuirk, Hidden-sector current-current correlators in holographic gauge mediation, Phys. Rev. D 85 (2012) 045025 [arXiv:1110.5075] [INSPIRE].

[44] F. Benini et al., Holographic gauge mediation, JHEP 12 (2009) 031 [arXiv:0903.0619] [INSPIRE].

[45] P. McGuirk, Falling flavors in AdS/CFT, JHEP 07 (2013) 102 [arXiv:1212.2210] [INSPIRE].

[46] K. Hashimoto and S. Nagaoka, Recombination of intersecting D-branes by local tachyon condensation, JHEP 06 (2003) 034 [hep-th/0303204] [INSPIRE].

[47] S. Nagaoka, Higher dimensional recombination of intersecting D-branes, JHEP 02 (2004) 063 [hep-th/0312010] [INSPIRE].

[48] S. Cecotti, M.C. Cheng, J.J. Heckman and C. Vafa, Yukawa couplings in F-theory and non-commutative geometry, arXiv:0910.0477 [INSPIRE].

[49] F. Marchesano, P. McGuirk and G. Shiu, Chiral matter wavefunctions in warped compactifications, JHEP 05 (2011) 090 [arXiv: 1012.2759] [INSPIRE].

[50] M. Mariño, R. Minasian, G.W. Moore and A. Strominger, Nonlinear instantons from supersymmetric p-branes, JHEP 01 (2000) 005 [hep-th/9911206] [INSPIRE].

[51] J. Gomis, F. Marchesano and D. Mateos, An open string landscape, JHEP 11 (2005) 021 [hep-th/0506179] [INSPIRE].

[52] L. Martucci and P. Smyth, Supersymmetric D-branes and calibrations on general $N=1$ backgrounds, JHEP 11 (2005) 048 [hep-th/0507099] [INSPIRE].

[53] D. Marolf, L. Martucci and P.J. Silva, Fermions, T duality and effective actions for D-branes in bosonic backgrounds, JHEP 04 (2003) 051 [hep-th/0303209] [INSPIRE].

[54] D. Marolf, L. Martucci and P.J. Silva, Actions and fermionic symmetries for D-branes in bosonic backgrounds, JHEP 07 (2003) 019 [hep-th/0306066] [INSPIRE].

[55] L. Martucci, J. Rosseel, D. Van den Bleeken and A. Van Proeyen, Dirac actions for D-branes on backgrounds with fluxes, Class. Quant. Grav. 22 (2005) 2745 [hep-th/0504041] [INSPIRE].

[56] M. Berkooz, M.R. Douglas and R.G. Leigh, Branes intersecting at angles, Nucl. Phys. B 480 (1996) 265 [hep-th/9606139] [INSPIRE]. 
[57] L. Aparicio, A. Font, L.E. Ibáñez and F. Marchesano, Flux and instanton effects in local F-theory models and hierarchical fermion masses, JHEP 08 (2011) 152 [arXiv:1104.2609] [INSPIRE].

[58] D. Cremades, L. Ibáñez and F. Marchesano, Computing Yukawa couplings from magnetized extra dimensions, JHEP 05 (2004) 079 [hep-th/0404229] [INSPIRE].

[59] F. Marchesano, P. McGuirk and G. Shiu, Open string wavefunctions in warped compactifications, JHEP 04 (2009) 095 [arXiv:0812.2247] [INSPIRE].

[60] R.C. Myers, Dielectric branes, JHEP 12 (1999) 022 [hep-th/9910053] [INSPIRE].

[61] B. Wynants, Supersymmetric actions for multiple D-branes on D-brane backgrounds, M.'s thesis, Katholieke Universiteit Leuven, Belgium (2006).

[62] J.P. Boyd, Chebyshev and Fourier spectral methods, Dover Publications Inc., U.S.A. (2001).

[63] E. Shuster, Killing spinors and supersymmetry on AdS, Nucl. Phys. B 554 (1999) 198 [hep-th/9902129] [INSPIRE].

[64] A. Chodos and E. Myers, Gravitational contribution to the Casimir energy in Kaluza-Klein theories, Annals Phys. 156 (1984) 412 [InSPIRE].

[65] K. Skenderis, Lecture notes on holographic renormalization, Class. Quant. Grav. 19 (2002) 5849 [hep-th/0209067] [INSPIRE].

[66] M. Krasnitz, Correlation functions in a cascading $N=1$ gauge theory from supergravity, JHEP 12 (2002) 048 [hep-th/0209163] [INSPIRE].

[67] O. Aharony, A. Buchel and A. Yarom, Holographic renormalization of cascading gauge theories, Phys. Rev. D 72 (2005) 066003 [hep-th/0506002] [INSPIRE].

[68] I.R. Klebanov and A.A. Tseytlin, Gravity duals of supersymmetric $\mathrm{SU}(N) \times \mathrm{SU}(N+M)$ gauge theories, Nucl. Phys. B 578 (2000) 123 [hep-th/0002159] [INSPIRE].

[69] M. Berg, M. Haack and W. Mueck, Glueballs vs. gluinoballs: fluctuation spectra in non-AdS/non-CFT, Nucl. Phys. B 789 (2008) 1 [hep-th/0612224] [INSPIRE].

[70] J. Polchinski, String theory. Volume 2: superstring theory and beyond, Cambridge University Press, Cambridge U.K. (1998).

[71] A.V. Meremianin, Hyperspherical harmonics with arbitrary arguments, J. Math. Phys. 50 (2009) 013526 [arXiv:0807.2128]. 